\title{
Communication \\ Nickel-Catalyzed Kumada Cross-Coupling Reactions of Benzylic Sulfonamides
}

\author{
Kirsten A. Hewitt, Claire A. Herbert, Alissa C. Matus (D) and Elizabeth R. Jarvo *(D) \\ Department of Chemistry, University of California, Irvine, CA 92697-2025, USA; khewitt1@uci.edu (K.A.H.); \\ caherber@uci.edu (C.A.H.); matusa@uci.edu (A.C.M.) \\ * Correspondence: erjarvo@uci.edu
}

Citation: Hewitt, K.A.; Herbert, C.A.;

Matus, A.C.; Jarvo, E.R.

Nickel-Catalyzed Kumada

Cross-Coupling Reactions of Benzylic Sulfonamides. Molecules 2021, 26 , 5947. https://doi.org/10.3390/ molecules 26195947

Academic Editors: William D. Lubell, Bradley L. Merner and Simon Giroux

Received: 2 September 2021

Accepted: 25 September 2021

Published: 30 September 2021

Publisher's Note: MDPI stays neutral with regard to jurisdictional claims in published maps and institutional affiliations.

\begin{abstract}
Herein, we report a Kumada cross-coupling reaction of benzylic sulfonamides. The scope of the transformation includes acyclic and cyclic sulfonamide precursors that cleanly produce highly substituted acyclic fragments. Preliminary data are consistent with a stereospecific mechanism that allows for a diastereoselective reaction.
\end{abstract}

Keywords: cross-coupling reactions; sulfonamides; nickel; catalysis; hydrocarbons

\section{Introduction}

Transition-metal catalyzed cross-coupling $(\mathrm{XC})$ reactions have transformed modern synthetic organic chemistry by creating an arsenal of carbon-carbon bond forming reactions [1-6]. Nickel is a cost-effective metal that is capable of activating challenging electrophiles such as amine derivatives [7-10]. Intense research efforts have been employed in the development of nickel-catalyzed XC reactions of sluggish electrophiles [11-13]. However, the $\mathrm{XC}$ reaction of alkyl amine derivatives has remained a significant challenge [14-18]. Historically, in order to facilitate nickel-catalyzed reactions, activation of these carbon-nitrogen bonds has been achieved via incorporation into strained aziridine rings or transformation to ammonium salts [19].

Ring-strain-promoted XC of aziridines has been accomplished [20]. Early stoichiometric work by Hillhouse established that aziridines undergo facile oxidative addition with nickel complexes [21]. Catalytic Negishi reactions of sulfonylaziridines have subsequently been established. The Doyle laboratory reported a regioselective Negishi XC reaction of styrenyl aziridines with alkylzinc reagents with substitution at the benzylic position (Scheme 1a) [22,23]. Key to their success was the use of an electron deficient fumarate ligand. Shortly thereafter, the Doyle and Jamison groups independently described a regioselective Negishi XC reaction of alkyl aziridines with alkylzinc reagents to forge the desired carbon-carbon bond (Scheme 1b,c) [24,25]. The differing regioselectivity of these reactions can be explained by comparing the oxidative addition events of the $\mathrm{C}-\mathrm{N}$ bonds. Styrenyl aziridines preferentially undergo oxidative addition at the benzylic center to afford a $\eta^{3}$-benzylnickel complex. In contrast, alkyl aziridines, which do not contain an aromatic ring to direct the nickel complex, preferentially undergo oxidative addition at the less hindered position [21]. These reports demonstrate the ability to activate the $\mathrm{C}-\mathrm{N}$ bond in strained rings.

Development of XC reactions of acyclic benzylamine derivatives has relied upon formation of highly reactive electrophiles (i.e., charged ammonium salts) [26,27]. For example, the Watson laboratory demonstrated that benzylic trimethylammonium salts are competent electrophiles in Suzuki-Miyaura XC reactions with aryl and vinylboronic acids (Scheme 2a) [28,29]. Similarly, the Wang laboratory disclosed the XC reaction of benzylic trimethylammonium salts with organoaluminum reagents to forge the desired carbon-carbon bond (Scheme 2b) [30]. 
a. Doyle (2012)

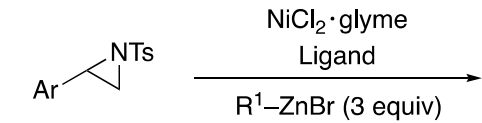

b. Doyle (2013)

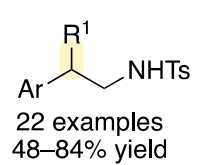

$48-84 \%$ yield $\underbrace{26-80 \% \text { yield }}_{26 \text { examples }}$

c. Jamison (2014)

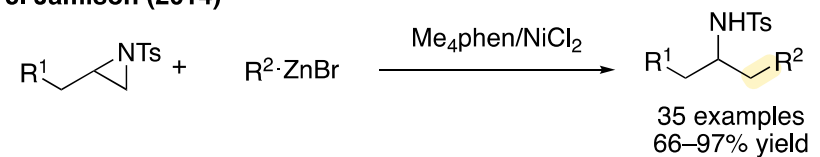<smiles>COC(=O)/C=C/C(=O)OC</smiles><smiles>CC(=O)/C=C/c1ccccc1S(=O)(=O)O</smiles>

Scheme 1. Cross-Coupling (XC) Reactions of Aziridines.

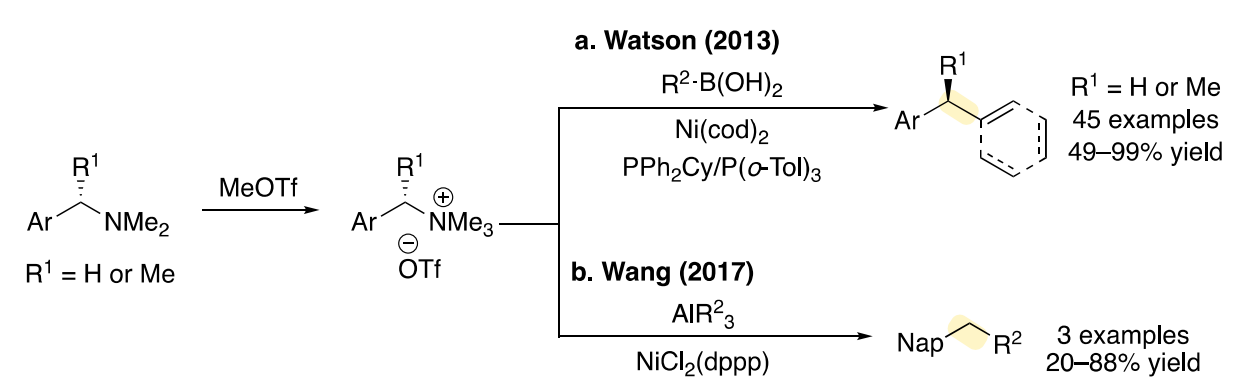

Scheme 2. XC Reactions of Trimethylammonium salts. Nap = Naphthyl.

The use of Katritzky salts to activate amines has proven to be sufficient for activation of benzylic and alkyl amines for Suzuki-Miyaura and Negishi XC reactions. Previously, it has been observed that Katritzky salts participate in $\mathrm{S}_{\mathrm{N}} 2$, radical, and Minisci-type reactions, and in recent years, many transition-metal catalyzed reactions have been developed [31-40]. The Watson laboratory hypothesized that these air and moisture stable salts would be suitable electrophiles in a XC reaction [41]. To test their hypothesis, primary amines were converted to Katritzky salts via a condensation reaction with 2,4,6-triphenylpyrylium tetrafluoroborate and the corresponding salts were subjected to Suzuki- Miyaura XC reactions with aryl boronic acids. The desired cross-coupled products were obtained in good yields (Scheme 3a) [42]. This strategy was amenable to the coupling of primary benzylic Katritzky salts as well (Scheme 3b) [43]. Additionally, vinyl boranes and alkylborane reagents, generated in situ by hydroboration of alkenes, participated in XC with Katritzky salts (Scheme 3c,d) [44,45]. This strategy has been extended beyond Suzuki-Miyaura reactions to include Negishi XC reactions with alkylzinc reagents (Scheme 3e) [46].

These methods establish strain- and charge-based strategies to activate amines for use as the electrophilic partner in XC reactions; however, the requirement for aziridines or functionalization as highly reactive ammonium salts remains a major limitation in broad application of these methods. In this manuscript, we report the first nickel-catalyzed Kumada XC reaction of simple benzylic sulfonamides with methylmagnesium iodide (Scheme 4). Previously, the Jarvo laboratory disclosed the Kumada XC reaction of benzylic ethers which proceeded in excellent yields, and enantio- and diastereoselectivity $[12,47,48]$. Building on this work, we aimed to develop an analogous reaction that employed benzylic sulfonamides. Ethers and sulfonamides have similar leaving group abilities, as the conjugate bases have similar $\mathrm{pK}_{\mathrm{a}}{ }^{\prime} \mathrm{s}$, and we hypothesized sulfonamides would behave similarly to ethers in a XC reaction $[49,50]$. In addition, these moieties are appealing because they are common functional groups in synthesis. Furthermore, we demonstrate that sulfonamides undergo stereospecific $X C$ reactions, in contrast to the stereoablative reactivity typically observed with styrenyl aziridines and Katritzky salts [22,36-40,51-54]. This stereospecific 
manifold allows for rapid diastereoselective construction of acyclic fragments bearing 1,3-substitution $[55,56]$.

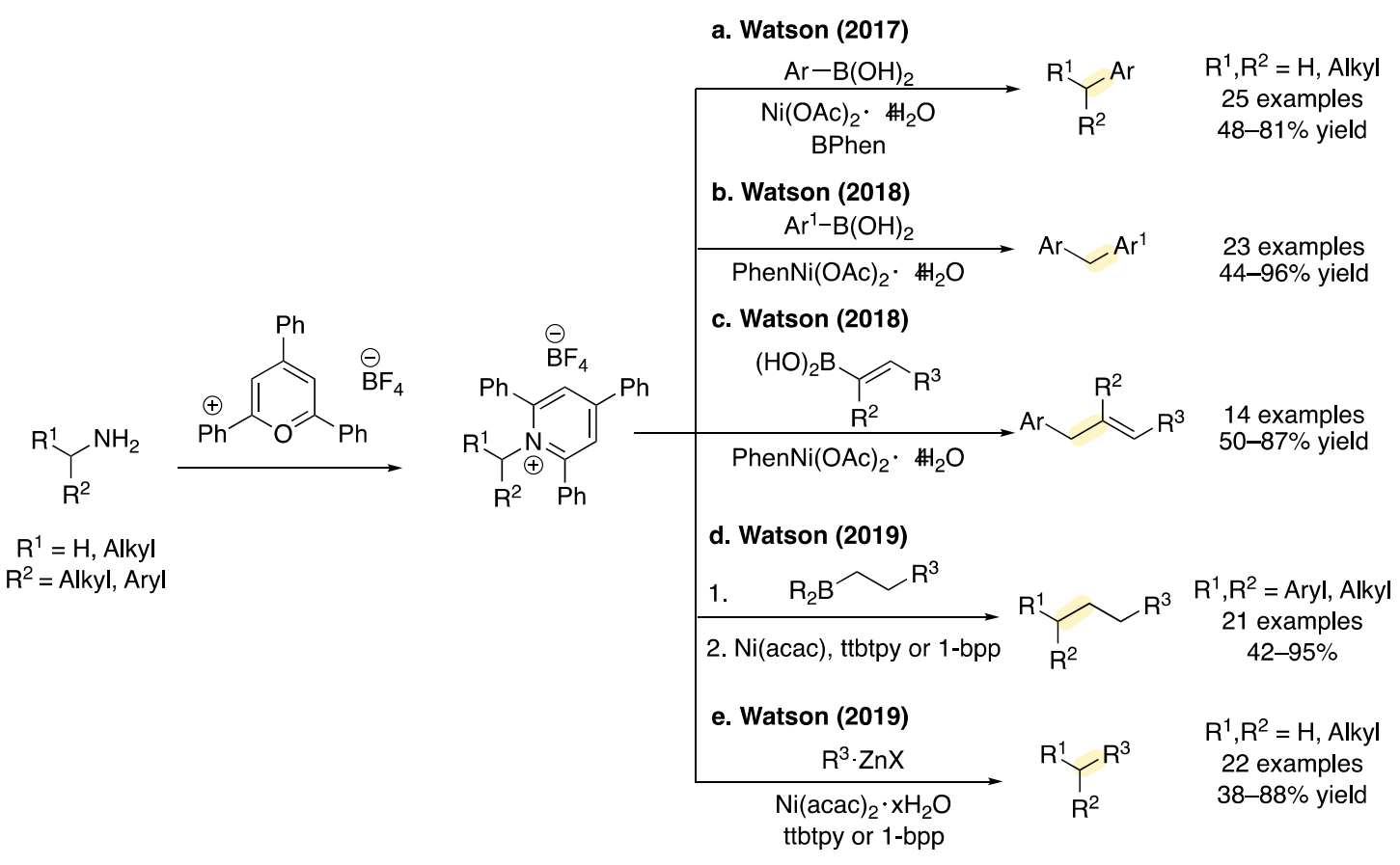

Scheme 3. XC Reactions of Katritzky Salts.

This Work

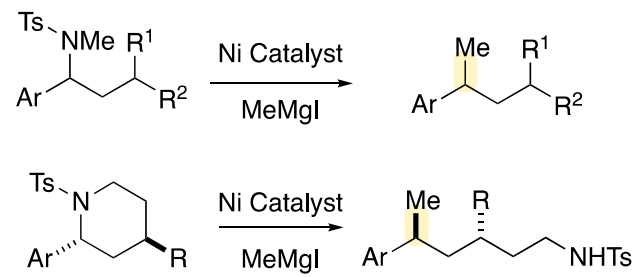

Scheme 4. Kumada XC Reactions of Benzylic Sulfonamides.

\section{Results and Discussion}

We began our investigation into the Kumada XC reaction with benzylic sulfonamide 1, which was synthesized in three steps from the commercially available aldehyde (See Experimental Section for substrate synthesis). Previously, Kumada XC reactions of benzylic ethers employed $\mathrm{Ni}(\mathrm{cod})_{2}$ and racemic BINAP as the optimal reaction conditions $[12,47,48]$. Under these conditions, we were excited to observe $25 \%$ yield of the desired cross-coupled product 2 (Table 1, entry 1). Increasing the catalyst loading to $15 \mathrm{~mol} \%$ improved the yield of the reaction (entry 2). However, it also increased the yield of the undesired styrene product 3 arising from $\beta$-hydride elimination. In an effort to improve the ratio between desired product 2 and styrene product 3 , we investigated a series of bidentate phosphine, $\mathrm{NHC}$, and pyridine ligands. DPEPhos improved the yield of $\mathbf{2}$ and decreased the amount of styrene $\mathbf{3}$ (entry 3). However, all other ligands evaluated did not improve the yield of 2 (entries 4-7). 
Table 1. Optimization of Kumada XC Reaction of Benzylic Sulfonamides.
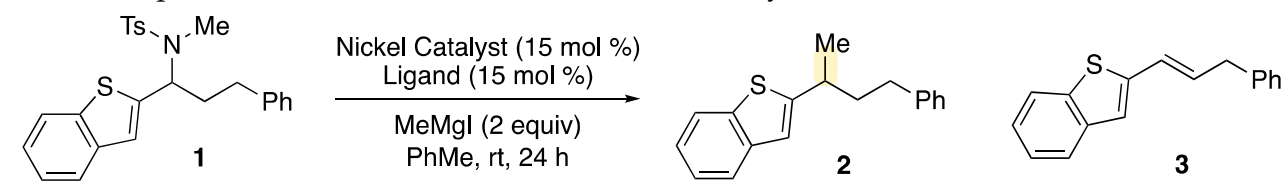

\begin{tabular}{cccccc}
\hline Entry & Nickel Catalyst & Ligand & Yield 2 (\%) & Yield 3 (\%) $^{\mathbf{1}}$ & RSM 1 (\%) $\mathbf{1}^{\mathbf{1}}$ \\
\hline $1^{2}$ & $\mathrm{Ni}(\operatorname{cod})_{2}$ & rac-BINAP & 25 & 10 & 19 \\
2 & $\mathrm{Ni}(\operatorname{cod})_{2}$ & rac-BINAP & 34 & 30 & 7 \\
3 & $\mathrm{Ni}(\operatorname{cod})_{2}$ & DPEPhos & 42 & 20 & 0 \\
4 & $\mathrm{Ni}(\operatorname{cod})_{2}$ & XantPhos & 0 & $<5$ & 37 \\
5 & $\mathrm{Ni}(\operatorname{cod})_{2}$ & dppe & 0 & $<5$ & 65 \\
6 & $\mathrm{Ni}(\operatorname{cod})_{2}$ & SiMes·BF & 12 & 0 & 86 \\
7 & $\mathrm{Ni}(\operatorname{cod})_{2}$ & BPhen & 0 & 0 & 61 \\
$\mathbf{8}$ & $\mathbf{( R - B I N A P ) N i C l} \mathbf{2}_{\mathbf{2}}$ & - & $\mathbf{5 4}$ & $\mathbf{4 0}$ & $\mathbf{0}$ \\
\hline
\end{tabular}

${ }^{1}$ Yield of $\mathbf{2}$ and $\mathbf{3}$ and Recovered Starting Material (RSM) determined by ${ }^{1} \mathrm{H}$ NMR based on comparison to PhTMS as internal standard. ${ }^{2} 5 \mathrm{~mol} \% \mathrm{Ni}(\mathrm{cod})_{2}$.

We next investigated an alternative precatalyst. Previously, the Jarvo laboratory reported the cross-electrophile coupling (XEC) reaction of benzylic and allylic sulfonamides which employed a BINAP-ligated nickel (II) precatalyst $[50,57,58]$. Utilizing these conditions, with $15 \mathrm{~mol} \%$ of catalyst, we were delighted to observe the desired product in $54 \%$ yield and $40 \%$ yield of styrene 3 (entry 8 ). We elected to proceed with the nickel (II) precatalyst as it provided the desired product in the highest yield.

With optimized conditions in hand, we evaluated the scope of the Kumada XC reaction (Scheme 5). Naphthyl substrates were well tolerated under the standard reaction conditions and product $\mathbf{4}$ was observed in $84 \%$ yield. Notably, products such as $\mathbf{5}$ and $\mathbf{6}$ with branching at the $\beta$-position provided good yields of cross-coupled products with lesser amounts of styrenes formed from $\beta$-hydride elimination $(20-30 \%)$ when compared to product 2 . We hypothesized that this increase in steric bulk destabilized the conformation necessary for $\beta$-hydride elimination to proceed.

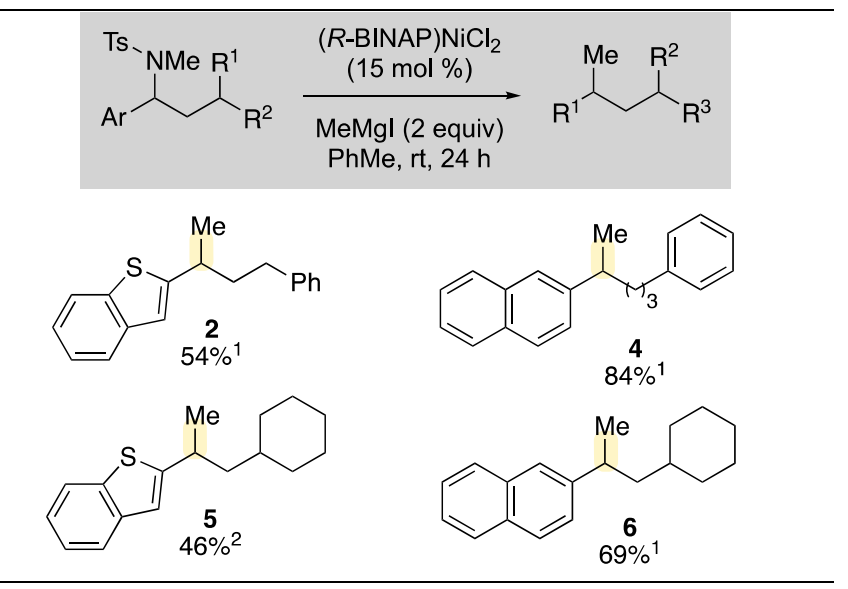

Scheme 5. Scope of the Kumada XC Reaction of Acyclic Sulfonamides. ${ }^{1}$ Yield determined by ${ }^{1} \mathrm{H}$ NMR based on comparison to PhTMS as internal standard. ${ }^{2}$ Isolated yield.

We also sought to evaluate a series of arylpiperidines, with the expectation that a stereospecific $\mathrm{XC}$ reaction at the benzylic position would provide synthetic access to highly substituted acyclic fragments. Furthermore, products would bear a pendant sulfonamide moiety, available for subsequent functionalization [50]. Rapid synthesis of the requisite cyclic sulfonamides was achieved by hetero Diels-Alder (HDA) cycloadditions or azaPrins reactions [59-61]. For substrates with alkyl substituents in the 4-position, [4+2] HDA reactions provided the requisite starting materials (Scheme 6a). For substrates 
bearing ether groups in the 4-position, an aza-Prins reaction provided the requisite 2-aryl4-hydroxylpiperidine that could be subsequently methylated or benzylated. (Scheme $6 \mathrm{~b}$ ).

a. Hetero Diels-Alder Reaction

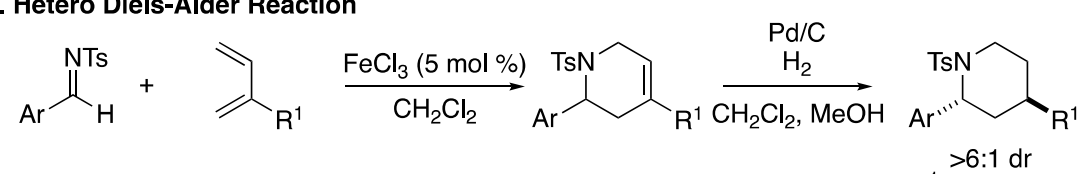

b. Aza-Prins Reaction $\mathrm{R}^{1}=\mathrm{Ph}$ or $\mathrm{Me}$

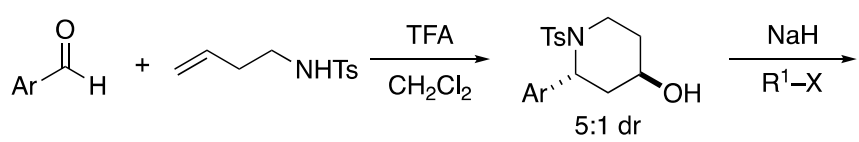

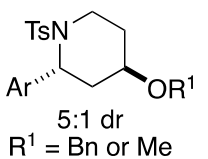

Scheme 6. Arylpiperidine Synthesis via (a) Hetero Diels-Alder (HDA) Reaction and (b) Aza-Prins Reaction.

With rapid and diastereoselective access to the desired piperidines, we examined these cyclic substrates in ring-opening Kumada XC reactions (Scheme 7). Phenyl and methyl substituents (products 7 and 8 ) were well tolerated and minimal amounts $(<5 \%)$ of $\beta$ hydride elimination were observed. Methylated and benzylated ethers were well tolerated and provided the desired products in good yields $(\mathbf{9}, \mathbf{1 0}$, and 11) [62]. It is important to note that the diastereomeric ratio observed in the products is consistent with the diastereomeric ratio of the starting material (See Materials and Methods Section). Therefore, preliminary data support a stereospecific Kumada XC reaction.

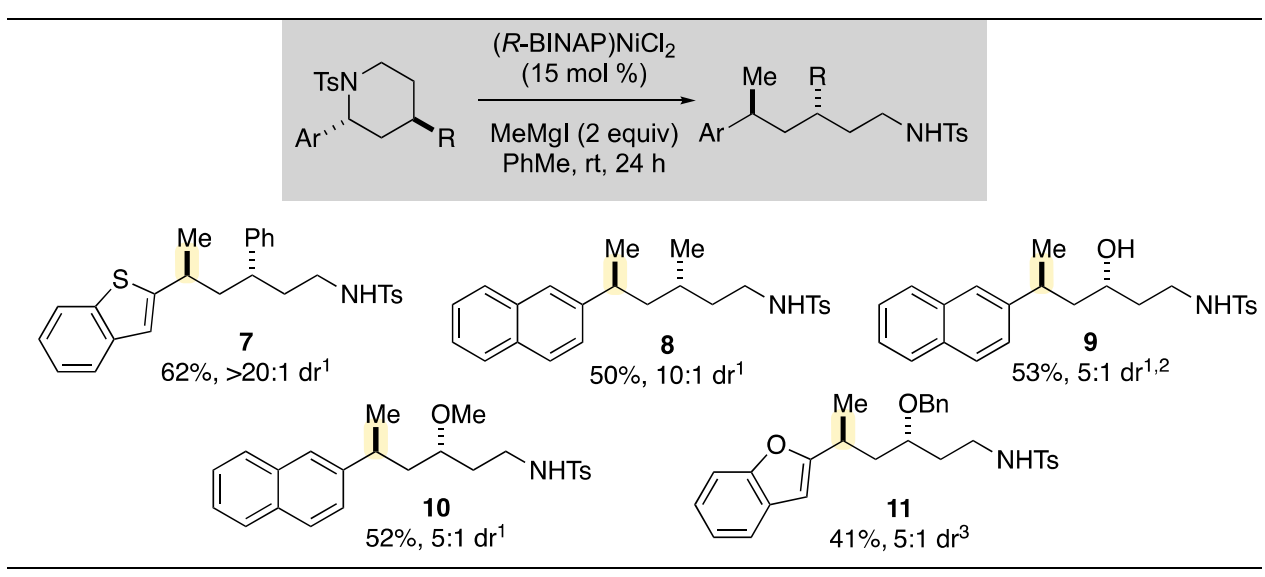

Scheme 7. Scope of the Kumada XC Reaction of Cyclic Sulfonamides. ${ }^{1}$ Isolated yield. ${ }^{2} \mathrm{R}=\mathrm{OBn}$ in starting material and provided the free alcohol in product. ${ }^{3}$ Yield determined by ${ }^{1} \mathrm{H}$ NMR based on comparison to PhTMS as internal standard.

To further develop the potential scope of this reaction, we sought to establish a ring-opening of a sulfonyl piperidine with an aryl Grignard reagent (Scheme 8). Such transformations would provide synthetic access to diarylalkanes bearing pendant sulfonamides, including rapid assembly of stereochemically-rich analogs of ATPase inhibitor 14 [63-67]. We have previously observed that in Kumada XC reactions of benzylic ethers employing aryl Grignard reagents, the optimal nickel catalyst is ligated by dppe [68]. We were pleased to see that this trend applied to benzylic sulfonamides: employing the commercially available precatalyst, (dppe) $\mathrm{NiCl}_{2}$, the XC reaction proceeded smoothly to provide the desired product 13 in 58\% isolated yield [69]. 

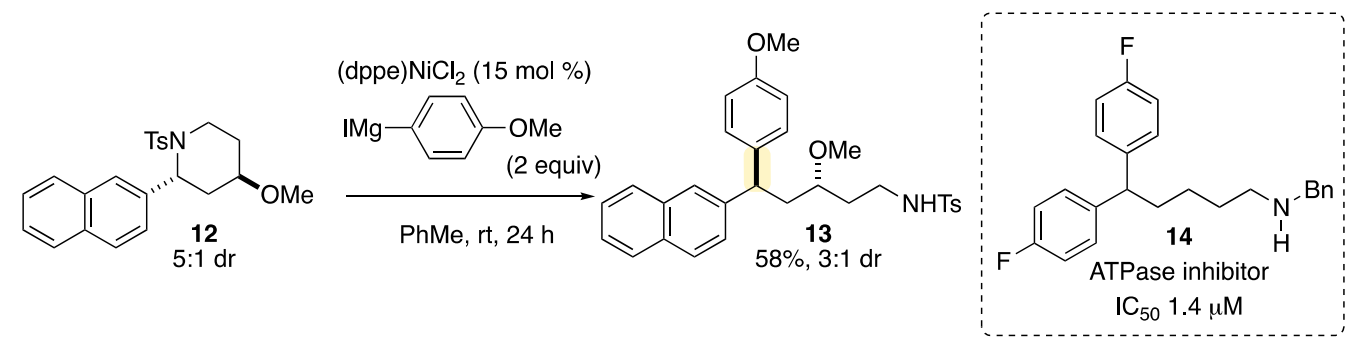

Scheme 8. Kumada XC Reaction with Aryl Grignard Reagent.

We propose the following catalytic cycle for the Kumada $\mathrm{XC}$ reaction based on related mechanisms for the Kumada XC reaction of benzylic ethers and the XEC reaction of benzylic sulfonamides (Scheme 9) [50,70]. First, reduction of the nickel(II) precatalyst with the Grignard reagent provides the active $\mathrm{Ni}(0)$ catalyst 15 . Next, oxidative addition of the benzylic sulfonamide affords the Ni(II) intermediate 16. Based on the calculated reaction coordinate diagram and transition state energies for related transformations, we hypothesize that rate-determining oxidative addition occurs with inversion of the benzylic carbon $[12,47,48,70]$. This step is facilitated by Lewis acidic magnesium salts that activate the sulfonamide moiety. Transmetallation with the Grignard reagent provides alkylnickel complex 17. Subsequent reductive elimination, which occurs with retention at the benzylic center, affords the desired product and turns over the catalyst. Alternatively, intermediate 16 can undergo $\beta$-hydride elimination to afford the observed styrene by-product.

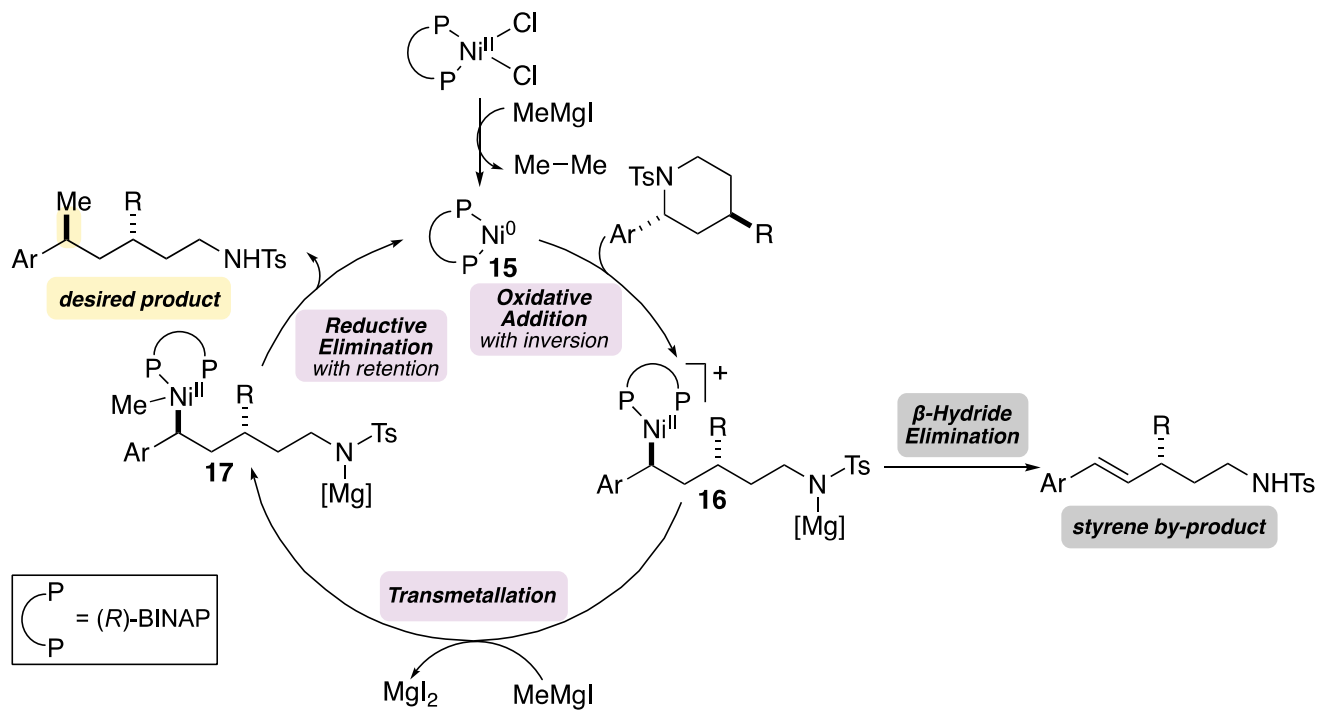

Scheme 9. Proposed Mechanism of Kumada XC Reaction. Speciation of magnesium complexes are omitted for clarity.

\section{Materials and Methods}

\subsection{General Procedures}

All reactions were carried out under an atmosphere of $\mathrm{N}_{2}$, or Ar when noted. All glassware was oven- or flame-dried prior to use. Tetrahydrofuran (THF), diethyl ether $\left(\mathrm{Et}_{2} \mathrm{O}\right)$, dichloromethane $\left(\mathrm{CH}_{2} \mathrm{Cl}_{2}\right)$, and toluene $(\mathrm{PhMe})$ were degassed with $\mathrm{Ar}$ and then passed through two $4 \times 36$ inch columns of anhydrous neutral A-2 alumina $(8 \times 14$ mesh; LaRoche Chemicals; activated under a flow of argon at $350{ }^{\circ} \mathrm{C}$ for $12 \mathrm{~h}$ ) to remove $\mathrm{H}_{2} \mathrm{O}$ [71]. All other solvents utilized were purchased anhydrous commercially, or purified as described. ${ }^{1} \mathrm{H}$ NMR spectra were recorded on Bruker DRX-400 $\left(400 \mathrm{MHz}{ }^{1} \mathrm{H}, 100 \mathrm{MHz}{ }^{13} \mathrm{C}\right.$, $\left.376.5 \mathrm{MHz}{ }^{19} \mathrm{~F}\right), \mathrm{GN}-500\left(500 \mathrm{MHz}{ }^{1} \mathrm{H}, 125.4 \mathrm{MHz}{ }^{13} \mathrm{C}\right)$, or CRYO-500 $\left(500 \mathrm{MHz}{ }^{1} \mathrm{H}\right.$, $\left.125.8 \mathrm{MHz}{ }^{13} \mathrm{C}\right)$ spectrometers. Proton chemical shifts are reported in ppm $(\delta)$ relative to internal tetramethylsilane (TMS, $\delta 0.00$ ). Data are reported as follows: chemical shift 
(multiplicity [singlet (s), broad singlet (br s), doublet (d), doublet of doublet (dd), doublet of doublet of doublets (ddd), doublet of doublet of doublet of doublets (dddd), doublet of triplet $(\mathrm{dt})$, doublet of doublet of triplet (ddt), doublet of triplet of doublet (dtd), triplet $(t)$, broad triplet (br $t$ ), triplet of doublet (td), triplet of doublet of doublet (tdd), triplet of triplet (tt), quartet (q), quartet of doublet (qd), quartet of doublet of doublets (qdd), quintet (quint), apparent quintet (appar quint), sextet, apparent sextet (appar sextet), multiplet (m)]. coupling constants [Hz], integration). Carbon chemical shifts are reported in ppm ( $\delta)$ relative to TMS with the respective solvent resonance as the internal standard $\left(\mathrm{CDCl}_{3}\right.$, $\delta 77.16 \mathrm{ppm})$. Unless otherwise indicated, NMR data were collected at $25^{\circ} \mathrm{C}$. Infrared (IR) spectra were obtained on a Thermo Scientific Nicolet iS5 spectrometer with an iD5 ATR tip (neat) and are reported in terms of frequency of absorption $\left(\mathrm{cm}^{-1}\right)$. Analytical thin-layer chromatography (TLC) was performed using Silica Gel $60 \mathrm{~F}_{254}$ precoated plates ( $0.25 \mathrm{~mm}$ thickness). Visualization was accomplished by irradiation with a UV lamp and/or staining with $\mathrm{KMnO}_{4}$ or CAM. Flash chromatography was performed using SiliaFlash F60 (40-63 $\mu \mathrm{m}, 60 \AA$ A) from SiliCycle. Automated chromatography was carried out on a Teledyne Isco CombiFlash Rf Plus. Melting points (m.p.) were obtained using a Mel-Temp melting point apparatus and are uncorrected. High resolution mass spectrometry was performed by the University of California, Irvine Mass Spectrometry Center. See the ${ }^{1} \mathrm{H}$, ${ }^{13} \mathrm{C}, \mathrm{COSY}$ and NOE NMR detailed data in the Supplementary Materials.

Bis(1,5-cyclooctadiene)nickel was purchased from Strem, stored in a glove box freezer $\left(-20^{\circ} \mathrm{C}\right)$ under an atmosphere of $\mathrm{N}_{2}$ and used as received. All ligands were purchased from Strem or Sigma Aldrich and were stored in a glovebox and used as received. The methylmagnesium iodide was titrated with iodine prior to use [72]. All other chemicals were purchased commercially and used as received, unless otherwise noted.

\subsection{Experimental}

\subsubsection{General Kumada Cross-Coupling Reaction Procedures}

Method A: Kumada Cross-Coupling Reaction

In a glovebox, a flame-dried $7 \mathrm{~mL}$ vial equipped with a stir bar was charged with sulfonamide substrate (1.0 equiv), nickel precatalyst $(15 \mathrm{~mol} \%)$ and PhMe $(0.10-0.20 \mathrm{M}$ in substrate). The Grignard reagent (2.0 equiv) was then added dropwise via a syringe. After $24 \mathrm{~h}$, the reaction was removed from the glovebox, quenched with methanol, filtered through a plug of silica gel eluting with $100 \% \mathrm{Et}_{2} \mathrm{O}$ and concentrated in vacuo. Phenyltrimethylsilane (PhTMS; $8.6 \mu \mathrm{L}, 0.050 \mathrm{mmol}$ ) was added and the yield was determined by ${ }^{1} \mathrm{H}$ NMR based on comparison to PhTMS as internal standard before purification by column chromatography.

For reactions in which 1.0 equiv of $\mathrm{MgI}_{2}$ is added, the vial is wrapped in aluminum foil for the duration of the reaction due to the light sensitivity of $\mathrm{MgI}_{2}$.

(1) Preparation of Grignard Reagent Under a $\mathrm{N}_{2}$ atmosphere, a three-necked flask equipped with a stir bar, reflux condenser, and Schlenk filtration apparatus was charged with magnesium turnings $(1.1 \mathrm{~g}, 45 \mathrm{mmol})$. The flask and magnesium turnings were then flame-dried under vacuum and the flask was back-filled with $\mathrm{N}_{2}$. Anhydrous $\mathrm{Et}_{2} \mathrm{O}(7.0 \mathrm{~mL}$ ) and a crystal of iodine (ca. $2.0 \mathrm{mg}$ ) were added to the flask. Freshly distilled iodomethane $(1.9 \mathrm{~mL}, 31 \mathrm{mmol})$ or 4-iodoanisole as a solution in $\mathrm{Et}_{2} \mathrm{O}$ $\left(4.7 \mathrm{~g}\right.$, 20. mmol, 6.7 $\mathrm{M}$ in $\left.\mathrm{Et}_{2} \mathrm{O}\right)$ was slowly added over $30 \mathrm{~min}$ to maintain a gentle reflux. The mixture was stirred for $2 \mathrm{~h}$ at room temperature then filtered through the fritted Schlenk filter into a Schlenk flask under $\mathrm{N}_{2}$ atmosphere. The magnesium turnings were washed with $\mathrm{Et}_{2} \mathrm{O}(2 \times 1.0 \mathrm{~mL})$ then the Schlenk flask was sealed, removed, and placed under an $\mathrm{N}_{2}$ atmosphere. The resulting methylmagnesium iodide was typically between 2.4 and 3.0 M as titrated by Knochel's method [72] and could be stored, sealed under $\mathrm{N}_{2}$ atmosphere or in a glovebox, for up to 4 weeks.

(2) Preparation of $(\boldsymbol{R}-\mathbf{B I N A P}) \mathbf{N i C l}_{\mathbf{2}}$ This method was adapted from a procedure reported by Jamison [57]. To a flame-dried $50 \mathrm{~mL}$ round bottom flask equipped with a stir bar was added $\mathrm{NiCl}_{2} \cdot 6 \mathrm{H}_{2} \mathrm{O}(0.24 \mathrm{~g}, 1.0 \mathrm{mmol}, 1.0$ equiv $)$. The flask was placed 
under vacuum and flame-dried until nearly all of the nickel compound had turned from emerald green to yellow-orange. Some of the green hexahydrate is necessary for the reaction to proceed. The flask was allowed to cool to room temperature then ( $R$-BINAP) ( $0.62 \mathrm{~g}, 1.0 \mathrm{mmol}, 1.0$ equiv) was added. The flask was then equipped with a reflux condenser and was evacuated and backfilled with $\mathrm{N}_{2}$. Then the solids were dissolved in $\mathrm{MeCN}(20 \mathrm{~mL}, 0.05 \mathrm{M})$ and the reaction mixture was allowed to reflux for $24 \mathrm{~h}$. Upon completion, the reaction was cooled to room temperature and the black crystalline precipitate was filtered under vacuum to yield a fine black powder $(0.53 \mathrm{~g}$, $0.71 \mathrm{mmol}, 71 \%$ yield).

\subsubsection{Characterization Data for Kumada Cross-Coupled Products}<smiles>CC(CC[PH+]c1ccccc1)c1cc2ccccc2s1</smiles>

2-(4-Phenylbutan-2-yl)benzo[b]thiophene (2) was prepared according to Method A. The following amounts of reagents were used: sulfonamide 1 ( $87 \mathrm{mg}, 0.20 \mathrm{mmol}, 1.0$ equiv), ( $R$ BINAP) $\mathrm{NiCl}_{2}(23 \mathrm{mg}, 30 . \mu \mathrm{mol}, 15 \mathrm{~mol} \%)$, PhMe (1.0 mL, $\left.0.20 \mathrm{M}\right)$, and methylmagnesium iodide $\left(0.16 \mathrm{~mL}, 0.40 \mathrm{mmol}, 2.0\right.$ equiv, $2.5 \mathrm{M}$ in $\left.\mathrm{Et}_{2} \mathrm{O}\right)$. Before purification, a ${ }^{1} \mathrm{H}$ NMR yield of $54 \%$ was obtained containing $40 \%$ styrene 3 based on comparison to PhTMS as an internal standard. The residue was purified by flash chromatography (0-5\% EtOAc/hexanes) to yield a mixture of the title compound and styrene 3 . To separate the major product and the styrene, an Upjohn dihydroxylation was performed [60,61]. The following amounts of reagents were used: substrate $\left(30 \mathrm{mg}, 0.12 \mathrm{mmol}, 1.0\right.$ equiv), $\mathrm{OsO}_{4}(7.6 \mu \mathrm{L}, 1.2 \mu \mathrm{mol}$, $1.0 \mathrm{~mol} \%, 4 \%$ solution in $\mathrm{H}_{2} \mathrm{O}$ ), $N$-methylmorpholine $N$-oxide (NMO) (16 mg, $0.13 \mathrm{mmol}$, 1.1 equiv), acetone $(0.25 \mathrm{~mL})$ and $\mathrm{H}_{2} \mathrm{O}(0.05 \mathrm{~mL})$. The residue was purified by flash column chromatography to afford the title compound as a colorless oil. (13 mg, $48 \mu \mathrm{mol}, 24 \%$ yield over two steps) with a small amount of styrene 3 (1.1 mg, $4.5 \mu \mathrm{mol}, 2.2 \%$ yield). TLC $\mathrm{R}_{\mathrm{f}}=0.8(5 \%$ EtOAc/hexanes $) ;{ }^{1} \mathrm{H}$ NMR $\left(500 \mathrm{MHz}, \mathrm{CDCl}_{3}\right) \delta 7.78(\mathrm{~d}, J=7.9 \mathrm{~Hz}, 1 \mathrm{H})$, $7.68(\mathrm{~d}, J=7.9 \mathrm{~Hz}, 1 \mathrm{H}), 7.45-7.22(\mathrm{~m}, 4 \mathrm{H}), 7.22-7.12(\mathrm{~m}, 3 \mathrm{H}), 7.04(\mathrm{~s}, 1 \mathrm{H}), 3.12$ (sextet, $J=7.0 \mathrm{~Hz}, 1 \mathrm{H}), 2.73-2.50(\mathrm{~m}, 2 \mathrm{H}), 2.12-1.92(\mathrm{~m}, 2 \mathrm{H}), 1.41(\mathrm{~d}, J=7.0 \mathrm{~Hz}, 3 \mathrm{H}) ;{ }^{13} \mathrm{C}$ NMR $\left(125.7 \mathrm{MHz}_{\mathrm{CDCl}}\right) \delta$ 152.5, 142.1, 140.0, 139.0, 128.5 (2C), 128.4 (2C), 125.8, 124.1, 123.5, $122.9,122.3,119.5,40.5,35.8,33.6,23.1$; IR (neat) 2927, 1456, 904, $726 \mathrm{~cm}^{-1}$; HRMS (TOF MS $\mathrm{ES}+$ ) $\mathrm{m} / z:[\mathrm{M}+\mathrm{Na}]^{+}$calcd. for $\mathrm{C}_{18} \mathrm{H}_{18} \mathrm{SNa} 289.1027$, found 289.1024 .<smiles>CC(CCCc1ccccc1)c1ccc2ccccc2c1</smiles>

2-(5-Phenylpentan-2-yl)naphthalene (4) was prepared according to Method A. The following amounts of reagents were used: sulfonamide 19 (22 mg, 50. $\mu$ mol, 1.0 equiv), (R-BINAP)NiCl 2 (5.6 mg, 7.5. $\mu \mathrm{mol}, 15 \mathrm{~mol} \%)$, PhMe $(0.25 \mathrm{~mL}, 0.20 \mathrm{M})$, and methylmagnesium iodide (40. $\mu \mathrm{L}, 0.10 \mathrm{mmol}, 2.0$ equiv, $2.8 \mathrm{M}_{\text {in }} \mathrm{Et}_{2} \mathrm{O}$ ). Before purification, a ${ }^{1} \mathrm{H}$ NMR yield of $84 \%$ was obtained containing $13 \%$ styrene 18 based on comparison to PhTMS as an internal standard. The residue was purified by flash chromatography (100\% hexanes) to yield the title compound as yellow oil (10. $\mathrm{mg}, 36 \mu \mathrm{mol}, 74 \%$ yield) containing styrene $18(1.3 \mathrm{mg}, 5.0 \mu \mathrm{mol}, 10 \%)$ and $\mathrm{CH}_{2} \mathrm{Cl}_{2}(0.8 \mathrm{mg}, 9.4 \mu \mathrm{mol}, 19 \%)$. TLC $\mathrm{R}_{\mathrm{f}}=0.8(5 \%$ EtOAc/hexanes); ${ }^{1} \mathrm{H}$ NMR (500 MHz, $\left.\mathrm{CDCl}_{3}\right) \delta 7.81-7.74(\mathrm{~m}, 3 \mathrm{H}), 7.60-7.55(\mathrm{~s}, 1 \mathrm{H}), 7.42$ (dddd, $J=16.3,8.2,6.8,1.4 \mathrm{~Hz}, 2 \mathrm{H}), 7.33(\mathrm{dd}, J=8.4,1.8 \mathrm{~Hz}, 1 \mathrm{H}), 7.23(\mathrm{~d}, J=7.5 \mathrm{~Hz}, 2 \mathrm{H})$, 7.17-7.09 (m, 3H), 2.88 (sextet, $J=7.0 \mathrm{~Hz}, 1 \mathrm{H}), 2.64-2.53(\mathrm{~m}, 2 \mathrm{H}), 1.79-1.55(\mathrm{~m}, 4 \mathrm{H}), 1.31(\mathrm{~d}$, $J=6.9 \mathrm{~Hz}, 3 \mathrm{H}) ;{ }^{13} \mathrm{C} \mathrm{NMR}\left(126 \mathrm{MHz}, \mathrm{CDCl}_{3}\right) \delta 145.2,142.7,133.8,132.3,128.5(2 \mathrm{C}), 128.4$ (2C), 128.0, 127.7, 127.7, 125.9, 125.9, 125.8, 125.3, 125.2, 40.2, 38.0, 36.1, 29.7, 22.5; HRMS (TOF MS Cl+) m/z: [M] $]^{+}$calcd. for $\mathrm{C}_{21} \mathrm{H}_{22} 274.1721$, found 274.1710. 


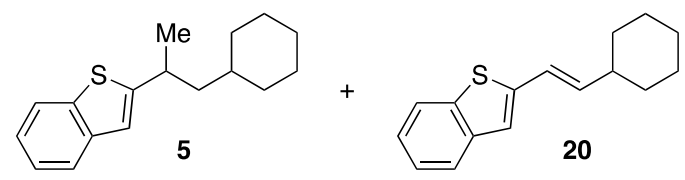

2-(1-Cyclohexylpropan-2-yl)benzo[b]thiophene (5) was prepared according to Method A. The following amounts of reagents were used: sulfonamide 21 ( $43 \mathrm{mg}, 0.10 \mathrm{mmol}, 1.0$ equiv), ( $R$-BINAP) $\mathrm{NiCl}_{2}(11 \mathrm{mg}, 15 \mu \mathrm{mol}, 15 \mathrm{~mol} \%)$, PhMe $(0.50 \mathrm{~mL}, 0.20 \mathrm{M})$, and methylmagne-

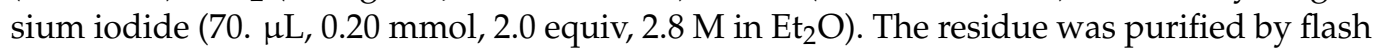
column chromatography (0-15\% $\mathrm{Et}_{2} \mathrm{O}$ /pentanes) to afford the title compound as a clear, colorless oil (12 mg, $46 \mu \mathrm{mol}, 46 \%$ yield) containing styrene $20(7.3 \mathrm{mg}, 30 . \mu \mathrm{mol}, 30 \%)$ and minimal amounts of solvent. TLC $\mathrm{R}_{\mathrm{f}}=0.7(100 \%$ pentanes $) ;{ }^{1} \mathrm{H}$ NMR: $\left(600 \mathrm{MHz}, \mathrm{CDCl}_{3}\right) \delta$ $7.83(\mathrm{~d}, J=7.2 \mathrm{~Hz}, 1 \mathrm{H}), 7.72(\mathrm{~d}, J=7.8 \mathrm{~Hz}, 1 \mathrm{H}), 7.30(\mathrm{td}, J=7.6,1.2 \mathrm{~Hz}, 1 \mathrm{H}), 7.26-7.22(\mathrm{~m}$, $1 \mathrm{H}), 7.00(\mathrm{~s}, 1 \mathrm{H}), 3.27$ (sextet, $J=12.6 \mathrm{~Hz}, 1 \mathrm{H}), 1.84-1.76(\mathrm{~m}, 1 \mathrm{H}), 1.70-1.57(\mathrm{~m}, 5 \mathrm{H}), 1.53-$ $1.44(\mathrm{~m}, 1 \mathrm{H}), 1.34(\mathrm{~d}, J=6.9 \mathrm{~Hz}, 3 \mathrm{H}), 1.32-1.23(\mathrm{~m}, 2 \mathrm{H}), 1.19-1.11(\mathrm{~m}, 2 \mathrm{H}), 0.96-0.85(\mathrm{~m}$, 2H) ${ }^{13}{ }^{\mathrm{C}} \mathrm{NMR}\left(150.9 \mathrm{MHz}, \mathrm{CDCl}_{3}\right) \delta$ 153.6, 140.2, 138.9, 124.1, 123.4, 122.9, 122.4, 119.0, 66.0, $46.7,35.2,33.7,33.3,26.8,26.4,23.8,15.4$; HRMS (TOF MS CI+) $m / z$ [M]+ calcd. for $\mathrm{C}_{17} \mathrm{H}_{22} \mathrm{~S}$ 258.1442 , found 258.1453 .<smiles>O=[N+]([O-])C1CCCCC1</smiles><smiles>C(=C/C1CCCCC1)\c1ccc2ccccc2c1</smiles>

2-(1-Cyclohexylpropan-2-yl)naphthalene (6) was prepared according to Method A. The following amounts of reagents were used: sulfonamide 23 ( $44 \mathrm{mg}, 0.10 \mathrm{mmol}, 1.0$ equiv), ( $R$ BINAP) $\mathrm{NiCl}_{2}(11 \mathrm{mg}, 15 \mu \mathrm{mol}, 15 \mathrm{~mol} \%)$, PhMe $(0.50 \mathrm{~mL}, 0.20 \mathrm{M})$, and methylmagnesium iodide (70. $\mu \mathrm{L}, 0.20 \mathrm{mmol}, 2.0$ equiv, $2.9 \mathrm{M}$ in $\mathrm{Et}_{2} \mathrm{O}$ ). Before purification, a ${ }^{1} \mathrm{H}$ NMR yield of $69 \%$ was obtained with $22 \%$ styrene 22 based on comparison to PhTMS as an internal standard. The residue was purified by flash column chromatography $(0-20 \%$ EtOAc/hexanes) to afford the title compound as a colorless oil (14 mg, $54 \mu \mathrm{mol}, 54 \%$ yield) with a small amount of styrene $22(3.7 \mathrm{mg}, 15 \mu \mathrm{mol}, 15 \%$ yield $)$. TLC $\mathrm{R}_{\mathrm{f}}=0.7(100 \%$ hexanes); ${ }^{1} \mathrm{H}$ NMR $\left(500 \mathrm{MHz}, \mathrm{CDCl}_{3}\right) \delta$ 7.85-7.73 (m, 3H), $7.60(\mathrm{~s}, 1 \mathrm{H}), 7.47-7.38(\mathrm{~m}, 2 \mathrm{H})$, $7.35(\mathrm{dd}, J=8.4,1.8 \mathrm{~Hz}, 1 \mathrm{H}), 2.99$ (sextet, $J=6.8 \mathrm{~Hz}, 1 \mathrm{H}), 1.81(\mathrm{~d}, J=13.0 \mathrm{~Hz}, 1 \mathrm{H}), 1.63$ $(\mathrm{tdd}, J=14.2,8.1,5.0 \mathrm{~Hz}, 5 \mathrm{H}), 1.50-1.41(\mathrm{~m}, 1 \mathrm{H}), 1.28(\mathrm{~d}, J=6.9 \mathrm{~Hz}, 3 \mathrm{H}), 1.19-1.06(\mathrm{~m}, 4 \mathrm{H})$, $0.95-0.83(\mathrm{~m}, 2 \mathrm{H}) ;{ }^{13} \mathrm{C}$ NMR $(125.8 \mathrm{MHz}, \mathrm{CDCl} 3) \delta 145.8,133.8,132.3,128.0,127.72,127.68$, 126.0, 125.9, 125.2, 125.1, 46.3, 37.0, 35.3, 33.9, 33.5, 26.9, 26.4, 26.4, 23.1; HRMS (TOF MS $\mathrm{CI}+) \mathrm{m} / z$ : [M]+ calcd. for $\mathrm{C}_{19} \mathrm{H}_{24} 252.1878$, found 252.1868.

3.2.3. Characterization Data for Ring Opening Kumada Cross-Coupled Products<smiles>N#CCCC(CCCN)c1cc2ccccc2s1</smiles>

N-(5-(benzo[b]thiophen-2-yl)-3-phenylhexyl)-4-methylbenzenesulfonamide (7) was prepared according to Method A. The following amounts of reagents were used: piperidine 24 ( $38 \mathrm{mg}$, $80 \mu \mathrm{mol}, 1.0$ equiv), (R-BINAP) $\mathrm{NiCl}_{2}(9.0 \mathrm{mg}, 12 \mu \mathrm{mol}, 15 \mathrm{~mol} \%)$, methylmagnesium iodide

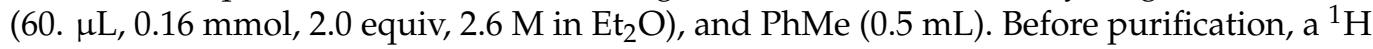
NMR yield of $64 \%$ was obtained. The residue was purified by column chromatography $(0-20 \%$ EtOAc/hexanes) to afford the title compound as pale yellow oil $(23 \mathrm{mg}, 49 \mu \mathrm{mol}$, $62 \%$ yield). The ratio of diastereomers was determined by integration of the resonances attributed to amine hydrogen in the ${ }^{1} \mathrm{H}$ NMR spectrum. The relative configuration of 7 was assigned based on analogy to a compound that has been previously reported $[12,47,48]$. $\mathrm{TLC} \mathrm{R}_{\mathrm{f}}=0.8(20 \% \mathrm{EtOAc} /$ hexanes$) ;{ }^{1} \mathrm{H} \mathrm{NMR}\left(400 \mathrm{MHz}, \mathrm{CDCl}_{3}\right) \delta 7.74(\mathrm{~d}, J=7.9 \mathrm{~Hz}, 1 \mathrm{H})$, 
7.67-7.56 (m, 3H), 7.37-7.13 (m, 9H), 7.07-7.00 (m, 2H), $6.93(\mathrm{~s}, 1 \mathrm{H}), 2.85(\mathrm{q}, \mathrm{J}=6.7 \mathrm{~Hz}$, $1 \mathrm{H}), 2.83-2.69(\mathrm{~m}, 2 \mathrm{H}), 2.70-2.59(\mathrm{~m}, 1 \mathrm{H}), 2.40(\mathrm{~s}, 3 \mathrm{H}), 2.07-1.95(\mathrm{~m}, 1 \mathrm{H}), 1.93-1.77(\mathrm{~m}, 2 \mathrm{H})$, 1.76-1.64 (m, 1H), $1.30(\mathrm{~d}, J=6.8 \mathrm{~Hz}, 3 \mathrm{H}) ;{ }^{13} \mathrm{C} \mathrm{NMR}\left(151 \mathrm{MHz}, \mathrm{CDCl}_{3}\right) \delta 152.7,143.5,143.4$, $140.0,138.9,136.9,129.9,129.8$ (2C), 128.9 (2C), 127.7, 127.6 (2C), 127.2 (2C), 126.8, 124.2, 123.6, 123.0, 122.3, 119.1, 45.8, 41.5, 40.9, 36.6, 33.4, 22.0, 21.7; HRMS (TOF MS ES+) $m / z$ $[\mathrm{M}+\mathrm{Na}]$ calcd. for $\mathrm{C}_{27} \mathrm{H}_{29} \mathrm{NO}_{2} \mathrm{~S}_{2} \mathrm{Na} 486.1537$, found 486.1524 .

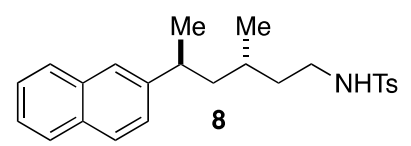

4-Methyl-N-(3-methyl-5-(naphthalen-2-yl)hexyl)benzenesulfonamide (8) was prepared according to Method A. The following amounts of reagents were used: piperidine 25 (10. $\mathrm{mg}$, 30. $\mu \mathrm{mol}, 1.0$ equiv), (R-BINAP) $\mathrm{NiCl}_{2}(3.0 \mathrm{mg}, 40 . \mu \mathrm{mol}, 15 \mathrm{~mol} \%)$, methylmagnesium iodide (10. $\mu \mathrm{L}, 60 . \mu \mathrm{mol}, 2.0$ equiv, $2.9 \mathrm{M}$ in $\left.\mathrm{Et}_{2} \mathrm{O}\right)$, and $\mathrm{PhMe}(0.30 \mathrm{~mL})$. Before purification, a ${ }^{1} \mathrm{H}$ NMR yield of $48 \%$ and 10:1 dr was obtained based on comparison to PhTMS as an internal standard. The residue was purified by flash column chromatography $(0-15 \%$ EtOAc/hexanes) to afford the title compound as a colorless oil (5.4 mg, $14 \mu \mathrm{mol}, 50 \%$ yield, $6: 1 \mathrm{dr})$ with a small amount of styrene $(0.6 \mathrm{mg}, 0.2 \mu \mathrm{mol}, 6 \%)$. The ratio of diastereomers was determined by integration of the resonances attributed to amine hydrogen in the ${ }^{1} \mathrm{H}$ NMR spectrum. The relative configuration of the major 8 was assigned based on analogy to ring opened compound 7. For clarity, the ${ }^{1} \mathrm{H}$ NMR and ${ }^{13} \mathrm{C}$ NMR data of the major and minor diastereomers have been tabulated individually.

TLC $R_{\mathrm{f}}=0.7$ (30\% EtOAc/hexanes, stained with CAM); HRMS (TOF MS E+) m/z [M+Na] calcd. for $\mathrm{C}_{24} \mathrm{H}_{29} \mathrm{NO}_{2} \mathrm{SNa}$, 418.1817; found, 418.1830 .

Major Diastereomer: ${ }^{1} \mathrm{H}$ NMR $\left(500 \mathrm{MHz}, \mathrm{CDCl}_{3}\right) \delta 7.81-7.76(\mathrm{~m}, 3 \mathrm{H}), 7.69(\mathrm{~d}, \mathrm{~J}=8.3$ $\mathrm{Hz}, 2 \mathrm{H}), 7.6(\mathrm{~s}, 1 \mathrm{H}), 7.47-7.40(\mathrm{~m}, 2 \mathrm{H}), 7.31-7.26(\mathrm{~m}, 3 \mathrm{H}), 4.29(\mathrm{t}, J=5.9 \mathrm{~Hz}, 1 \mathrm{H}), 3.03-2.82$ $(\mathrm{m}, 3 \mathrm{H}), 2.41(\mathrm{~s}, 3 \mathrm{H}), 1.49(\mathrm{t}, J=6.2 \mathrm{~Hz}, 1 \mathrm{H}), 1.43-1.46(\mathrm{~m}, 1 \mathrm{H}), 1.30-1.25(\mathrm{~m}, 3 \mathrm{H}), 1.25(\mathrm{~d}$, $J=6.9 \mathrm{~Hz}, 3 \mathrm{H}), 0.79(\mathrm{~d}, J=6.5 \mathrm{~Hz}, 3 \mathrm{H}) ;{ }^{13} \mathrm{C} \mathrm{NMR}\left(125.8, \mathrm{CDCl}_{3}\right) \delta 145.1,148.4,133.7,132.2$, 129.7 (2C), 128.1, 127.6, 127.6, 127.1 (2C), 126.0, 125.6, 125.2, 125.0, 45.6, 41.1, 37.2, 36.5, 28.1, 29.1, 22.1, 21.5, 19.6 .

Minor Diastereomer: ${ }^{1} \mathrm{H}$ NMR $\left(500 \mathrm{MHz}, \mathrm{CDCl}_{3}\right) \delta 7.81-7.76(\mathrm{~m}, 3 \mathrm{H}), 7.69(\mathrm{~d}, J=8.3$, 2H), $7.63(\mathrm{~s}, 1 \mathrm{H}), 7.47-7.40(\mathrm{~m}, 2 \mathrm{H}), 7.31-7.26(\mathrm{~m}, 3 \mathrm{H}), 4.39(\mathrm{t}, J=5.5 \mathrm{~Hz}, 1 \mathrm{H}), 3.03-2.82$ $(\mathrm{m}, 3 \mathrm{H}), 2.41(\mathrm{~s}, 3 \mathrm{H}), 1.49(\mathrm{t}, J=6.2 \mathrm{~Hz}, 1 \mathrm{H}), 1.43-1.46(\mathrm{~m}, 1 \mathrm{H}), 1.30-1.25(\mathrm{~m}, 3 \mathrm{H}), 1.25(\mathrm{~d}$, $J=6.9 \mathrm{~Hz}, 3 \mathrm{H}), 1.08(\mathrm{~d}, J=6.7,3 \mathrm{H}) ;{ }^{13} \mathrm{C} \mathrm{NMR}\left(125.8, \mathrm{CDCl}_{3}\right) \delta 145.1,148.4,133.7,132.2$, 129.7 (2C), 128.1, 127.6, 127.6, 127.1 (2C), 126.0, 125.7, 125.2, 125.0, 45.6, 41.5, 37.2, 36.5, 29.7, $29.1,22.1,21.5,19.6$.<smiles>CCCCCCCNCC[C@@H](O)CC(C)c1ccc2ccccc2c1</smiles>

N-(3-hydroxy-5-(naphthalen-2-yl)hexyl)-4-methylbenzenesulfonamide (9) was prepared according to Method A. The following amounts of reagents were used: piperidine 26 (93 mg, $0.20 \mathrm{mmol}, 1.0$ equiv), (R-BINAP) $\mathrm{NiCl}_{2}(23 \mathrm{mg}, 30 . \mu \mathrm{mol}, 15 \mathrm{~mol} \%)$, methylmagnesium iodide $\left(0.14 \mathrm{~mL}, 0.40 \mathrm{mmol}, 2.0\right.$ equiv, $2.9 \mathrm{M}$ in $\left.\mathrm{Et}_{2} \mathrm{O}\right)$, and PhMe $(2.0 \mathrm{~mL})$. The residue was purified by flash column chromatography (0-15\% EtOAc/hexanes) to afford the title compound as a colorless oil ( $42 \mathrm{mg}, 0.11 \mathrm{mmol}, 53 \%$ yield, 5:1 dr). The ratio of diastereomers was determined by integration of the resonances attributed to amine hydrogen in the ${ }^{1} \mathrm{H}$ NMR spectrum. The relative configuration of the major 9 was assigned based on analogy to ring opened compound 7. For clarity, the ${ }^{1} \mathrm{H}$ NMR and ${ }^{13} \mathrm{C}$ NMR data of the major and minor diastereomers have been tabulated individually.

TLC $R_{\mathrm{f}}=0.5$ (30\% EtOAc/hexanes, stained with CAM); HRMS (TOF MS E+) m/z $[\mathrm{M}+\mathrm{Na}]$ calcd. for $\mathrm{C}_{23} \mathrm{H}_{27} \mathrm{NO}_{3} \mathrm{SNa}, 420.1609$; found, 420.1604 . 
Major Diastereomer: ${ }^{1} \mathrm{H}$ NMR $\left(500 \mathrm{MHz}, \mathrm{CDCl}_{3}\right) \delta 7.79-7.75(\mathrm{~m}, 3 \mathrm{H}), 7.69(\mathrm{~d}$, $J=8.15 \mathrm{~Hz}, 2 \mathrm{H}), 7.59(\mathrm{~s}, 1 \mathrm{H}), 7.43$ (appar quint, $J=7.44 \mathrm{~Hz}, 2 \mathrm{H}), 7.31(\mathrm{~d}, J=8.82 \mathrm{~Hz}$, $1 \mathrm{H}), 7.23(\mathrm{~d}, J=8.17 \mathrm{~Hz}, 2 \mathrm{H}), 5.27(\mathrm{t}, J=5.55 \mathrm{~Hz}, 1 \mathrm{H}), 3.74-3.70(\mathrm{~m}, 1 \mathrm{H}), 3.12-3.06(\mathrm{~m}$, $1 \mathrm{H}), 3.01-2.93(\mathrm{~m}, 2 \mathrm{H}), 2.37(\mathrm{~s}, 3 \mathrm{H}), 1.89(\mathrm{br} \mathrm{s}, 1 \mathrm{H}), 1.86-1.80(\mathrm{~m}, 1 \mathrm{H}), 1.72-1.62(\mathrm{~m}, 2 \mathrm{H})$, 1.53-1.46 (m, $1 \mathrm{H}), 1.28(\mathrm{~d}, J=7.23 \mathrm{~Hz}, 3 \mathrm{H}) ;{ }^{13} \mathrm{C}$ NMR $\left(125.8, \mathrm{CDCl}_{3}\right) \delta 144.4,143.3,136.9$, $133.7,132.3,129.7$ (2C), 128.4, 127.7, 127.6, 127.1 (2C), 126.1, 125.5, 124.4, 125.0, 69.2, 45.9, $40.8,37.0,36.0,22.2,21.5$.

Minor Diastereomer: ${ }^{1} \mathrm{H}$ NMR $\left(500 \mathrm{MHz}, \mathrm{CDCl}_{3}\right) \delta 7.79-7.75(\mathrm{~m}, 3 \mathrm{H}), 7.65(\mathrm{~d}$, $J=8.14 \mathrm{~Hz}, 2 \mathrm{H}), 7.59(\mathrm{~s}, 1 \mathrm{H}), 7.43$ (appar quint, $J=7.44 \mathrm{~Hz}, 2 \mathrm{H}), 7.29(\mathrm{~d}, J=8.91 \mathrm{~Hz}$, $1 \mathrm{H}), 7.19(\mathrm{~d}, J=8.05 \mathrm{~Hz}, 2 \mathrm{H}), 5.19(\mathrm{t}, J=5.57 \mathrm{~Hz}, 1 \mathrm{H}), 3.43-3.39(\mathrm{~m}, 1 \mathrm{H}), 3.12-3.06(\mathrm{~m}$, $1 \mathrm{H}), 2.91-2.82(\mathrm{~m}, 2 \mathrm{H}), 2.35(\mathrm{~s}, 3 \mathrm{H}), 1.86-1.80(\mathrm{~m}, 1 \mathrm{H}), 1.76(\mathrm{br} \mathrm{s}, 1 \mathrm{H}), 1.72-1.62(\mathrm{~m}, 2 \mathrm{H})$, $1.53-1.46(\mathrm{~m}, 1 \mathrm{H}), 1.29(\mathrm{~d}, J=6.40 \mathrm{~Hz}, 3 \mathrm{H}) ;{ }^{13} \mathrm{C}$ NMR $\left(125.8, \mathrm{CDCl}_{3}\right) \delta 143.7,143.3,136.8$, $133.7,132.3,129.7$ (2C), 128.4, 127.7, 127.6, 127.1 (2C), 126.1, 125.5, 124.4, 125.0, 68.7, 45.6, $40.8,36.45,36.4223 .2,21.5$.

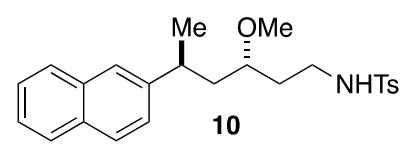

N-(3-methoxy-5-(naphthalen-2-yl)hexyl)-4-methylbenzenesulfonamide (10) was prepared according to Method A. The following amounts of reagents were used: piperidine 12 (58 $\mathrm{mg}$, $0.15 \mathrm{mmol}, 1.0$ equiv), ( $R$-BINAP) $\mathrm{NiCl}_{2}(15 \mathrm{mg}, 20 . \mu \mathrm{mol}, 15 \mathrm{~mol} \%)$, methylmagnesium

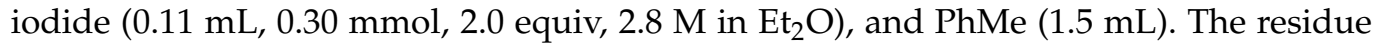
was purified by flash column chromatography $(0-25 \%$ EtOAc/hexanes) to afford the title compound as a colorless oil $(32 \mathrm{mg}, 80 . \mu \mathrm{mol}, 52 \%$ yield, $5: 1 \mathrm{dr})$. The ratio of diastereomers was determined by integration of the resonances attributed to amine hydrogen in the ${ }^{1} \mathrm{H}$ NMR spectrum. The relative configuration of the major $\mathbf{1 0}$ was assigned based on analogy to ring opened compound 7. For clarity, the ${ }^{1} \mathrm{H}$ NMR and ${ }^{13} \mathrm{C}$ NMR data of the major and minor diastereomers have been tabulated individually.

TLC $R_{\mathrm{f}}=0.4(30 \%$ EtOAc/hexanes, stained with CAM); HRMS (TOF MS E+) $\mathrm{m} / z[\mathrm{M}+$ $\mathrm{Na}$ ] calcd. for $\mathrm{C}_{24} \mathrm{H}_{29} \mathrm{NO}_{3} \mathrm{SNa}$, 424.1766; found, 434.1775.

Major Diastereomer: ${ }^{1} \mathrm{H}$ NMR $\left(500 \mathrm{MHz}, \mathrm{CDCl}_{3}\right) \delta 7.81-7.76(\mathrm{~m}, 3 \mathrm{H}), 7.68(\mathrm{~d}$, $J=8.37 \mathrm{~Hz}, 2 \mathrm{H}), 7.55(\mathrm{~s}, 1 \mathrm{H}), 7.47-7.40(\mathrm{~m}, 2 \mathrm{H}), 7.29(\mathrm{dd}, J=8.48,1.70 \mathrm{~Hz}, 1 \mathrm{H}), 7.26-$ $7.24(\mathrm{~m}, 1 \mathrm{H}), 7.21(\mathrm{~d}, J=7.97 \mathrm{~Hz}, 1 \mathrm{H}), 5.11(\mathrm{t}, J=5.53 \mathrm{~Hz}, 1 \mathrm{H}), 3.15(\mathrm{~s}, 3 \mathrm{H}), 3.08-2.91(\mathrm{~m}$, $3 \mathrm{H}), 2.86$ (appar sextet, $J=7.37 \mathrm{~Hz}, 1 \mathrm{H}), 2.34(\mathrm{~s}, 3 \mathrm{H}), 1.96(\mathrm{ddd}, J=14.3,8.50,5.78 \mathrm{~Hz}, 1 \mathrm{H})$, $1.82-1.76(\mathrm{~m}, 1 \mathrm{H}), 1.58-1.50(\mathrm{~m}, 2 \mathrm{H}), 1.28(\mathrm{~d}, J=6.98 \mathrm{~Hz}, 3 \mathrm{H}) ;{ }^{13} \mathrm{C} \mathrm{NMR}\left(125.8, \mathrm{CDCl}_{3}\right) \delta$ $144.1,143.2,136.8,133.6,132.3,129.7$ (2C), 128.3, 127.7, 127.6, 127.1 (2C), 126.1, 125.4, 125.24, 125.17 78.4, 56.3, 40.9, 40.5, 36.3, 32.0, 22.9, 21.5.

Minor Diastereomer: ${ }^{1} \mathrm{H}$ NMR $\left(500 \mathrm{MHz}, \mathrm{CDCl}_{3}\right) \delta 7.81-7.76(\mathrm{~m}, 3 \mathrm{H}), 7.70(\mathrm{~d}$, $J=8.60 \mathrm{~Hz}, 2 \mathrm{H}), 7.55(\mathrm{~s}, 1 \mathrm{H}), 7.47-7.40(\mathrm{~m}, 2 \mathrm{H}), 7.29(\mathrm{dd}, J=8.48,1.70 \mathrm{~Hz}, 1 \mathrm{H}), 7.26-$ $7.24(\mathrm{~m}, 1 \mathrm{H}), 7.21(\mathrm{~d}, J=7.97 \mathrm{~Hz}, 1 \mathrm{H}), 5.03(\mathrm{t}, J=5.52 \mathrm{~Hz}, 1 \mathrm{H}), 3.20(\mathrm{~s}, 3 \mathrm{H}), 3.08-2.91(\mathrm{~m}$, $3 \mathrm{H}), 2.86$ (appar sextet, $J=7.37 \mathrm{~Hz}, 1 \mathrm{H}), 2.38(\mathrm{~s}, 3 \mathrm{H}), 1.96(\mathrm{ddd}, J=14.3,8.50,5.78 \mathrm{~Hz}, 1 \mathrm{H})$, 1.71-1.66 (m, 1H), 1.47-1.40 (m, 2H), $1.27(\mathrm{~d}, J=7.32 \mathrm{~Hz}, 3 \mathrm{H}){ }^{13} \mathrm{C}$ NMR $\left(125.8, \mathrm{CDCl}_{3}\right)$ $\delta$ 144.2, 143.2, 136.9, 133.6, 132.3, 129.7 (2C), 128.3, 127.7, 127.6, 127.1 (2C), 126.1, 125.52, $125.46125 .4,78.4,56.3,40.9,40.5,36.3,32.0,22.9,21.5$.

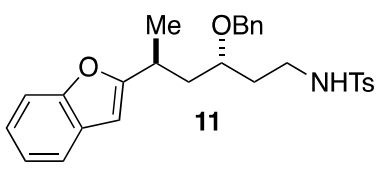

N-(5-(benzofuran-2-yl)-3-(benzyloxy)hexyl)-4-methylbenzenesulfonamide (11) was prepared according to Method A. The following amounts of reagents were used: piperidine 27 (78 mg, $0.17 \mathrm{mmol}, 1.0$ equiv), ( $R$-BINAP) $\mathrm{NiCl}_{2}(22 \mathrm{mg}, 30 . \mu \mathrm{mol}, 15 \mathrm{~mol} \%)$, methylmagnesium

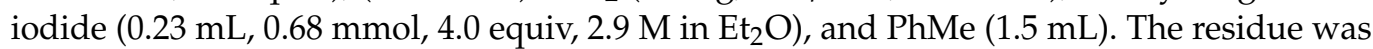


purified by flash column chromatography (0-15\% EtOAc/hexanes) to afford a mixture of the title compound and styrene. To separate the major product and the styrene, a dihydroxylation was performed. The following amounts of reagents were used: AD-mix- $\beta$ (52 $\mathrm{mg}, 1.4 \mathrm{~g} / \mathrm{mmol}), t-\mathrm{BuOH}(1.0 \mathrm{~mL})$, and $\mathrm{H}_{2} \mathrm{O}(1.0 \mathrm{~mL})$. The residue was purified by flash column chromatography to afford the title compound as a colorless oil. ( $7.0 \mathrm{mg}$, $15 \mu \mathrm{mol}, 8.6 \%$ yield over two steps, 5:1 dr). The ratio of diastereomers was determined by integration of the resonances attributed to amine hydrogen in the ${ }^{1} \mathrm{H}$ NMR spectrum. The relative configuration of the major $\mathbf{1 1}$ was assigned based on analogy to ring opened compound 7 . When the reaction was performed with 2.0 equivalents of methylmagnesium iodide, a ${ }^{1} \mathrm{H}$ NMR yield of $41 \%$ was obtained based on comparison to PhTMS as an internal standard before purification. For clarity, the ${ }^{1} \mathrm{H}$ NMR and ${ }^{13} \mathrm{C}$ NMR data of the major and minor diastereomers have been tabulated individually.

TLC $R_{\mathrm{f}}=0.5$ (30\% EtOAc/hexanes, stained with CAM); HRMS (TOF MS E+) $\mathrm{m} / \mathrm{z}$ $[\mathrm{M}+\mathrm{Na}]$ calcd. for $\mathrm{C}_{28} \mathrm{H}_{31} \mathrm{NO}_{4} \mathrm{SNa}$, 500.1872; found, 500.1861.

Major Diastereomer: ${ }^{1} \mathrm{H}$ NMR $\left(500 \mathrm{MHz}, \mathrm{CDCl}_{3}\right) \delta 7.64(\mathrm{~d}, J=7.9 \mathrm{~Hz}, 2 \mathrm{H}), 7.48(\mathrm{~d}$, $J=7.4 \mathrm{~Hz}, 1 \mathrm{H}), 7.42(\mathrm{~d}, J=7.9 \mathrm{~Hz}, 1 \mathrm{H}), 7.27-7.16(\mathrm{~m}, 8 \mathrm{H}), 6.33(\mathrm{~s}, 1 \mathrm{H}), 4.79(\mathrm{t}, J=6.0 \mathrm{~Hz}$, $1 \mathrm{H}), 4.45(\mathrm{~d}, J=11.5 \mathrm{~Hz}, 1 \mathrm{H}), 4.31(\mathrm{~d}, J=11.4 \mathrm{~Hz}, 1 \mathrm{H}), 3.49-3.42(\mathrm{~m}, 1 \mathrm{H}), 3.09-2.95(\mathrm{~m}, 3 \mathrm{H})$, $2.38(\mathrm{~s}, 3 \mathrm{H}), 2.12($ appar quint, $J=6.9 \mathrm{~Hz}, 1 \mathrm{H}), 1.87-1.81(\mathrm{~m}, 1 \mathrm{H}), 1.65-1.54(\mathrm{~m}, 3 \mathrm{H}), 1.29(\mathrm{~d}$, $J=6.9 \mathrm{~Hz}, 3 \mathrm{H}){ }^{13} \mathrm{C}$ NMR $\left(125.8, \mathrm{CDCl}_{3}\right) \delta 162.6,154.5,143.3,137.9,136.9,129.7$ (2C), 128.5 (2C), 128.1 (2C), 127.9, 127.1 (2C), 123.4, 122.6, 120.5, 110.9, 101.1, 75.0, 70.7, 40.2, 39.1, 32.9, 30.3, 29.7, 21.5, 19.7 .

Minor Diastereomer: ${ }^{1} \mathrm{H}$ NMR $\left(500 \mathrm{MHz}, \mathrm{CDCl}_{3}\right) \delta 7.80(\mathrm{~d}, J=7.9 \mathrm{~Hz}, 2 \mathrm{H}), 7.75(\mathrm{~d}$, $J=7.7 \mathrm{~Hz}, 1 \mathrm{H}), 7.58(\mathrm{~d}, J=8.4 \mathrm{~Hz}, 1 \mathrm{H}), 7.27-7.16(\mathrm{~m}, 8 \mathrm{H}), 6.25(\mathrm{~s}, 1 \mathrm{H}), 4.79(\mathrm{t}, J=6.0 \mathrm{~Hz}$, $1 \mathrm{H}), 4.40(\mathrm{~s}, 2 \mathrm{H}), 3.53-3.48(\mathrm{~m}, 1 \mathrm{H}), 3.09-2.95(\mathrm{~m}, 3 \mathrm{H}), 2.73$ (appar quint, $J=6.5 \mathrm{~Hz}, 1 \mathrm{H})$, $2.40(\mathrm{~s}, 3 \mathrm{H}), 1.80-1.77(\mathrm{~m}, 1 \mathrm{H}), 1.65-1.60(\mathrm{~m}, 3 \mathrm{H}), 0.88(\mathrm{~d}, J=7.0 \mathrm{~Hz}, 3 \mathrm{H}) ;{ }^{13} \mathrm{C}$ NMR $(125.8$, $\mathrm{CDCl}_{3}$ ) $\delta 162.6,154.5,143.3,137.9,136.9,129.4(2 \mathrm{C}), 128.7$ (2C), $128.3(2 \mathrm{C}), 127.9,127.1(2 \mathrm{C})$, $123.4,122.6,120.5,110.9,101.4,75.6,71.4,39.9,39.1,32.8,30.5,29.7,21.5,20.2$.<smiles>COc1ccc(C(CCCN)c2ccc3ccccc3c2)cc1</smiles>

N-3-methoxy-5-(4-methoxyphenyl)-5-(naphthalen-2-yl)pentyl)-4-methylbenzenesulfonamide (13) was prepared according to Method A. The following amounts of reagents were used: piperidine 12 (37 mg, $90 \mu \mathrm{mol}, 1.0$ equiv), $\mathrm{Ni}($ dppe $) \mathrm{Cl}_{2}(7.0 \mathrm{mg}, 10 . \mu \mathrm{mol}, 15 \mathrm{~mol} \%)$,

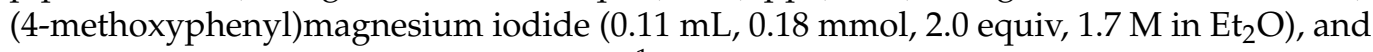
PhMe $(0.90 \mathrm{~mL})$. Before purification, a ${ }^{1} \mathrm{H}$ NMR yield of $56 \%$ was obtained based on comparison to PhTMS as an internal standard. The residue was purified by flash column chromatography (0-20\% EtOAc/hexanes) to afford the title compound as a yellow oil (26 mg, 50. $\mu \mathrm{mol}, 58 \%$ yield, 3:1 dr). The ratio of diastereomers was determined by integration of the resonances attributed to methyl hydrogens of the tosyl group in the ${ }^{1} \mathrm{H}$ NMR spectrum. The relative configuration of the major 13 was assigned based on analogy to ring opened compound 7. For clarity, the ${ }^{1} \mathrm{H}$ NMR and ${ }^{13} \mathrm{C}$ NMR data of the major and minor diastereomers have been tabulated individually.

TLC $R_{\mathrm{f}}=0.3$ (30\% EtOAc/hexanes, stained with CAM); HRMS (TOF MS E+) $\mathrm{m} / \mathrm{z}$ $[\mathrm{M}+\mathrm{H}]$ calcd. for $\mathrm{C}_{30} \mathrm{H}_{34} \mathrm{NO}_{4} \mathrm{~S}$, 504.2209; found, 504.2206.

Major Diastereomer: ${ }^{1} \mathrm{H}$ NMR $\left(500 \mathrm{MHz}, \mathrm{CDCl}_{3}\right) \delta 7.76(\mathrm{~d}, J=7.3 \mathrm{~Hz}, 2 \mathrm{H}), 7.70$ $7.69(\mathrm{~m}, 3 \mathrm{H}), 7.61(\mathrm{~s}, 1 \mathrm{H}), 7.43(\mathrm{dt}, J=20.4,7.3 \mathrm{~Hz}, 2 \mathrm{H}), 7.29-7.19(\mathrm{~m}, 3 \mathrm{H}), 7.13(\mathrm{~d}, J=8.5 \mathrm{~Hz}$, $2 \mathrm{H}), 6.81(\mathrm{~d}, J=8.6 \mathrm{~Hz}, 2 \mathrm{H}), 5.09-5 .-5(\mathrm{~m}, 1 \mathrm{H}), 4.12(\mathrm{t}, J=7.8 \mathrm{~Hz}, 1 \mathrm{H}), 3.76(\mathrm{~s}, 3 \mathrm{H}), 3.20(\mathrm{~s}$, $3 \mathrm{H}), 3.13-3.09(\mathrm{~m}, 1 \mathrm{H}), 3.05-2.97(\mathrm{~m}, 2 \mathrm{H}), 2.30(\mathrm{~s}, 3 \mathrm{H}), 2.25$ (appar sext, $J=7.3 \mathrm{~Hz}, 1 \mathrm{H})$, 2.02-1.97 (m, 1H), 1.85-1.80 (m, 1H), 1.58-1.52 (m, 1H); ${ }^{13} \mathrm{C} \mathrm{NMR}\left(125.8, \mathrm{CDCl}_{3}\right) \delta 158.2$, 
$143.4,142.4,136.8,135.2,133.6,129.7$ (2C), $129.0(2 \mathrm{C}), 128.8,128.3,127.8,127.7,127.2(2 \mathrm{C})$, $126.5,126.2,125.6,125.5,114.1(2 \mathrm{C}), 78.0,56.7,55.3,46.5,40.3,39.1,31.8,21.5$.

Minor Diastereomer: ${ }^{1} \mathrm{H}$ NMR $\left(500 \mathrm{MHz}, \mathrm{CDCl}_{3}\right) \delta 7.76(\mathrm{~d}, J=7.3 \mathrm{~Hz}, 2 \mathrm{H}), 7.73$ $7.70(\mathrm{~m}, 3 \mathrm{H}), 7.63(\mathrm{~s}, 1 \mathrm{H}), 7.43(\mathrm{dt}, J=20.4,7.3 \mathrm{~Hz}, 2 \mathrm{H}), 7.29-7.19(\mathrm{~m}, 3 \mathrm{H}), 7.14(\mathrm{~d}, J=7.7 \mathrm{~Hz}$, $2 \mathrm{H}), 6.81(\mathrm{~d}, J=8.8 \mathrm{~Hz}, 2 \mathrm{H}), 5.09-5 .-5(\mathrm{~m}, 1 \mathrm{H}), 4.12(\mathrm{t}, J=7.8 \mathrm{~Hz}, 1 \mathrm{H}), 3.76(\mathrm{~s}, 3 \mathrm{H}), 3.19(\mathrm{~s}$, $3 \mathrm{H}), 3.13-3.09(\mathrm{~m}, 1 \mathrm{H}), 3.05-2.97(\mathrm{~m}, 2 \mathrm{H}), 2.35(\mathrm{~s}, 3 \mathrm{H}), 2.25$ (appar sext, $J=7.3 \mathrm{~Hz}, 1 \mathrm{H})$, 2.06-2.03 (m, 1H), 1.76-1.78 (m, 1H), 1.58-1.52 (m, 1H); ${ }^{13} \mathrm{C}$ NMR $\left(125.8, \mathrm{CDCl}_{3}\right) \delta 158.2$, $143.4,142.0,136.9,136.5,133.6,129.7(2 \mathrm{C}), 129.0(2 \mathrm{C}), 128.8,128.4,127.8,127.7,127.2(2 \mathrm{C})$, $126.5,126.2,125.9,125.6,114.1$ (2C), 78.0, 56.7, 55.3, 46.5, 40.3, 39.1, 31.9, 21.6.

3.2.4. General Procedures for Synthesis of Starting Materials Method B: Condensation Reaction

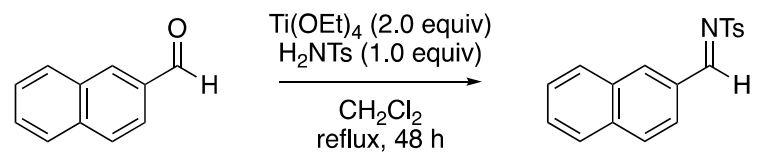

This method was adapted from a procedure reported by Ruano et al. [73]. A flame-dried two-neck flask equipped with a stir bar, condenser, septum and $\mathrm{N}_{2}$ inlet was charged with aldehyde (1.0 equiv), and $p$-toluenesulfonamide (1.0 equiv) and $\mathrm{CH}_{2} \mathrm{Cl}_{2}(330 \mathrm{~mL})$. Then $\mathrm{Ti}(\mathrm{OEt})_{4}(2.0$ equiv) was added dropwise. The deep orange solution was brought to reflux $\left(\sim 45^{\circ} \mathrm{C}\right)$ and allowed to stir for $48 \mathrm{~h}$. The solution was cooled to room temperature and was quenched with $\mathrm{H}_{2} \mathrm{O}$. The mixture was vacuum filtered and the filtrate was concentrated in vacuo.

Method C: Methylation of Sulfonamide with Methyl Iodide

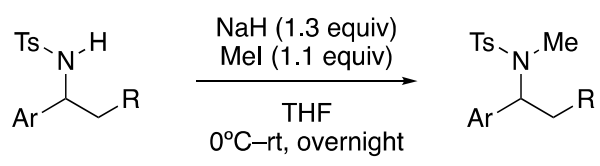

This method was adapted from a procedure reported by Jarvo [12,47,48]. To a suspension of $\mathrm{NaH}$ (1.3 equiv) in THF $(0.10 \mathrm{M})$ was added a solution of sulfonamide (1.0 equiv) in THF $(0.15 \mathrm{M})$ at $0{ }^{\circ} \mathrm{C}$. The mixture was warmed to rt and allow to stir for $1 \mathrm{~h}$ before the addition of iodomethane (1.1 equiv). The reaction was allowed to stir overnight at rt. The excess $\mathrm{NaH}$ was quenched with sat. $\mathrm{NH}_{4} \mathrm{Cl}$ and the solution was extracted with EtOAc $(\times 3)$. The combined organic layers were washed with brine, dried over $\mathrm{Na}_{2} \mathrm{SO}_{4}$, concentrated in vacuo, and purified by flash column chromatography.

Method D: Fe-Catalyzed Formal [4+2] Cycloaddition

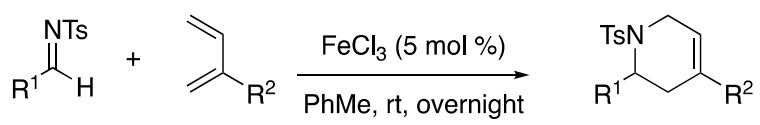

This method was adapted from a procedure reported by Matsubara [59]. To a flame-dried round-bottom flask equipped with a stir bar was added imine (1.0 equiv), $\mathrm{FeCl}_{3}(5.0 \mathrm{~mol} \%$ ), and $\mathrm{PhMe}(0.1 \mathrm{M})$. Once the solution was homogenous, diene (2.0 equiv) was added. The reaction mixture was allowed to stir at rt overnight. After completion, the reaction mixture was filtered through a short pad of silica, washed with excess ethyl acetate, and concentrated in vacuo.

Method E: Pd/C Reduction of Alkenes

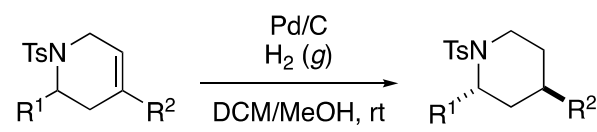


A flame-dried round-bottom flask with stir bar was charged with palladium on carbon (1.0 mg/3.5 mmol of substrate), flushed with $\mathrm{N}_{2}$, and capped with septum. Slowly, DCM was added, until $\mathrm{Pd} / \mathrm{C}$ was fully submerged. Then $\mathrm{MeOH}(0.2 \mathrm{M}$ in substrate), and alkene (1.0 equiv) were added. Vacuum was pulled on the flask until the solvent began to bubble, at which point the flask was backfilled with $\mathrm{N}_{2}(\times 3)$. An $\mathrm{H}_{2}$ balloon was added and the reaction mixture was allowed to stir vigorously until complete by ${ }^{1} \mathrm{H}$ NMR. The balloon was then removed, and the flask was purged with $\mathrm{N}_{2}$ for $30 \mathrm{~min}$. The septum was removed, and the reaction mixture was filtered through Celite using $\mathrm{MeOH}(100 \mathrm{~mL})$. The collected solvent was then concentrated in vacuo.

Method F: TFA Mediated Aza-Prins Cyclization

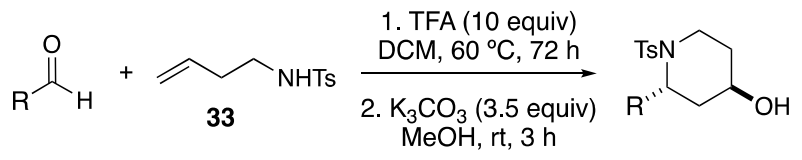

This method was adapted from a procedure reported by Sabitha [60,61]. To a flamedried pressure tube equipped with a stir bar was added aldehyde (1.0 equiv), homoallylic sulfonamide 33 (1.1 equiv), and $\mathrm{CH}_{2} \mathrm{Cl}_{2}(0.10 \mathrm{M})$. Then trifluoroacetic acid (10.0 equiv) was added slowly via syringe. The solution was warmed to $60^{\circ} \mathrm{C}$ and allowed to stir for $72 \mathrm{~h}$. The solution was then cooled to rt and quenched with saturated aq. $\mathrm{NaHCO}_{3}$. Then the $\mathrm{pH}$ was adjusted to $>7$ by the addition of $\mathrm{Et}_{3} \mathrm{~N}$. The solution was transferred to a separatory funnel, and the aqueous layer was extracted with $\mathrm{CH}_{2} \mathrm{Cl}_{2}(\times 3)$. The combined organic layers were washed with brine, dried over $\mathrm{Na}_{2} \mathrm{SO}_{4}$, filtered and concentrated in vacuo. The residue was then redissolved in $\mathrm{MeOH}$, and $\mathrm{K}_{2} \mathrm{CO}_{3}$ (3.5 equiv) was added to the flask and the slurry was allowed to stir at $\mathrm{rt}$ for $3 \mathrm{~h}$. The solvent was removed under reduced pressure, then $\mathrm{H}_{2} \mathrm{O}$ was added and the residue was transferred to a separatory funnel. The aqueous layer was extracted with $\mathrm{EtOAc}(\times 3)$. The combined organic layers were washed with brine, dried over $\mathrm{Na}_{2} \mathrm{SO}_{4}$, filtered and concentrated in vacuo.

Method G: Alkylation of Secondary Alcohol

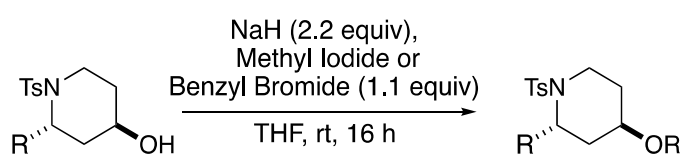

This method was adapted from a procedure reported by Yang [74]. In a glovebox, to a flame-dried round bottom flask equipped with a stir bar was added $\mathrm{NaH}$ (2.2 equiv). The flask was removed from the glovebox and $\mathrm{NaH}$ was dissolved in THF (0.2 M). Alcohol (1.0 equiv) was added dropwise as a solution in THF $(0.3 \mathrm{M})$ and the reaction mixture was allowed to stir at $\mathrm{rt}$ for $1 \mathrm{~h}$. Methyl iodide or benzyl bromide was then added dropwise to the stirring slurry and the reaction mixture was allowed to stir at $\mathrm{rt}$ overnight. The reaction was then quenched with saturated aq. $\mathrm{NH}_{4} \mathrm{Cl}$ and extracted with EtOAc $(\times 3)$. The combined organic layers were washed with brine, dried over $\mathrm{Na}_{2} \mathrm{SO}_{4}$ and concentrated in vacuo.

3.2.5. Synthesis and Characterization Data of Sulfonamide Starting Materials<smiles>[N+]=[N+]=Cc1cc2ccccc2s1</smiles>

$\mathrm{N}$-(benzo[b]thiophen-2-ylmethylene)-4-methylbenzenesulfonamide (28) was prepared according to Method B. The following amounts of reagents were used: benzo[b]thiophene2-carbaldehyde ( $3.2 \mathrm{~g}, 20$. mmol, 1.0 equiv), p-toluenesulfonamide $(3.1 \mathrm{~g}, 20 . \mathrm{mmol}$, 
1.0 equiv), $\mathrm{Ti}(\mathrm{OEt})_{4}\left(8.4 \mathrm{~mL}, 40 . \mathrm{mmol}, 2.0\right.$ equiv), and $\mathrm{CH}_{2} \mathrm{Cl}_{2}(330 \mathrm{~mL})$. The residue was purified by flash column chromatography (5-25\% EtOAc/hexanes) to yield the title compound as a pale yellow solid $(5.0 \mathrm{~g}, 16 \mathrm{mmol}, 80 \%)$. m.p. $148-150{ }^{\circ} \mathrm{C}$; $\mathrm{TLC} \mathrm{R}_{\mathrm{f}}=0.5$ (25\% EtOAc/hexanes); ${ }^{1} \mathrm{H}$ NMR $\left(500 \mathrm{MHz}, \mathrm{CDCl}_{3}\right) \delta 9.23(\mathrm{~s}, 1 \mathrm{H}), 8.02(\mathrm{~s}, 1 \mathrm{H}), 7.90(\mathrm{~d}$, $J=8.3,3 \mathrm{H}), 7.86(\mathrm{~d}, J=8.2,1 \mathrm{H}), 7.50(\mathrm{td}, J=8.3,1.2,1 \mathrm{H}), 7.42(\mathrm{td}, J=8.4,1.2,1 \mathrm{H}), 7.35(\mathrm{~d}$, $J=8.4,2 \mathrm{H}), 2.44(\mathrm{~s}, 3 \mathrm{H}) ;{ }^{13} \mathrm{C}$ NMR $\left(126 \mathrm{MHz}, \mathrm{CDCl}_{3}\right) \delta 163.3,144.8,143.6,138.9,138.3,137.3$, 135.3, 130.0 (2C), 128.7, 128.2 (2C), 126.1, 125.5, 123.2, 21.8; IR (neat) 3259, 2921, 1566, 1305, $1292,1152,1087,752 \mathrm{~cm}^{-1}$; HRMS (TOF MS ES+) $m / z$ calcd. for $\mathrm{C}_{16} \mathrm{H}_{13} \mathrm{NO}_{2} \mathrm{~S}_{2}[\mathrm{M}+\mathrm{Na}]^{+}$ 338.0285 , found 338.0283 .<smiles>[N+]=[N+]=Cc1ccc2ccccc2c1</smiles>

4-Methyl-N-(naphthalen-2-ylmethylene)benzenesulfonamide (29) was prepared according to Method B. The following amounts of reagents were used: 2-naphthaldehyde ( $0.31 \mathrm{~g}$, $2.0 \mathrm{mmol}, 1.0$ equiv), $p$-toluenesulfonamide $\left(0.34 \mathrm{~g}, 2.0 \mathrm{mmol}, 1.0\right.$ equiv), $\mathrm{Ti}(\mathrm{OEt})_{4}(0.59 \mathrm{~mL}$, $4.0 \mathrm{mmol}, 2.0$ equiv), and $\mathrm{CH}_{2} \mathrm{Cl}_{2}(33 \mathrm{~mL})$. The residue was purified by flash column chromatography (0-25\% EtOAc/hexanes) to yield the title compound as a pale yellow solid $(0.31 \mathrm{~g}, 1.0 \mathrm{mmol}, 50 \%$ yield $)$. Analytical data are consistent with literature values. [75] ${ }^{1} \mathrm{H}$ NMR $\left(400 \mathrm{MHz}, \mathrm{CDCl}_{3}\right) \delta 9.18(\mathrm{~s}, 1 \mathrm{H}), 8.34(\mathrm{~s}, 1 \mathrm{H}), 8.04(\mathrm{dd}, J=8.6,1.7 \mathrm{~Hz}, 1 \mathrm{H})$, 7.98-7.86 (m, 5H), 7.67-7.55 (m, 2H), 7.36 (d, J = 8.0 Hz, 2H), $2.44(\mathrm{~s}, 3 \mathrm{H})$.<smiles>O=[N+]([O-])C(CCc1ccccc1)c1cc2ccccc2s1</smiles>

$N-(1-(b e n z o[b]$ thiophen-2-yl)-3-phenylpropyl)-N,4-dimethylbenzenesulfonamide (1) was prepared according to Method $\mathrm{C}$. The following amounts of reagents were used: $\mathrm{N}-(1-$ (benzo[b]thiophen-2-yl)-3-phenylpropyl)-4-methylbenzenesulfonamide $(680 \mathrm{mg}, 1.6 \mathrm{mmol}$, 1.0 equiv), $\mathrm{NaH}$ (50. mg, $2.1 \mathrm{mmol}, 1.3$ equiv), methyl iodide $(0.11 \mathrm{~mL}, 1.8 \mathrm{mmol}, 1.1$ equiv) and THF $(30 \mathrm{~mL})$. The residue was purified by flash column chromatography $(5-25 \%$ EtOAc/hexanes) to yield the title compound as a yellow oil $(0.52 \mathrm{~g}, 1.2 \mathrm{mmol}, 68 \%$ yield $)$ $\mathrm{TLC} \mathrm{R}_{\mathrm{f}}=0.8\left(25 \%\right.$ EtOAc/hexanes); ${ }^{1} \mathrm{H}$ NMR $\left(500 \mathrm{MHz}, \mathrm{CDCl}_{3}\right) \delta 7.73(\mathrm{~d}, J=7.9 \mathrm{~Hz}, 1 \mathrm{H})$, 7.70-7.62 (m, 3H), 7.40-7.27 (m, 4H), 7.27-7.19 (m, 3H), $7.16(\mathrm{~d}, J=7.7 \mathrm{~Hz}, 2 \mathrm{H}), 7.06(\mathrm{~s}$, $1 \mathrm{H}), 5.42(\mathrm{t}, J=7.5 \mathrm{~Hz}, 1 \mathrm{H}), 2.77(\mathrm{~s}, 3 \mathrm{H}), 2.69(\mathrm{qdd}, J=14.1,10.4,5.9 \mathrm{~Hz}, 2 \mathrm{H}), 2.40(\mathrm{~s}, 3 \mathrm{H})$, 2.30 (dddd, $J=13.7,10.3,7.2,5.4 \mathrm{~Hz}, 1 \mathrm{H}), 2.08$ (dddd, $J=13.9,10.5,7.8,6.3 \mathrm{~Hz}, 1 \mathrm{H}) ;{ }^{13} \mathrm{C}$ NMR (125.7 MHz, $\left.\mathrm{CDCl}_{3}\right) \delta 143.31,143.30,141.0,139.6,139.2,137.0,129.6(2 \mathrm{C}), 128.6(2 \mathrm{C})$, 128.5 (2C), 127.3 (2C), 126.3, 124.5, 124.4, 123.6, 122.9, 122.3, 56.5, 34.6, 33.0, 29.0, 21.6; HRMS (TOF MS ES+) $\mathrm{m} / z$ : $[\mathrm{M}+\mathrm{Na}]^{+}$calcd. for $\mathrm{C}_{25} \mathrm{H}_{25} \mathrm{NO}_{2} \mathrm{~S}_{2} \mathrm{Na} 458.1224$, found 458.1235.<smiles>CN(C)C(CCCc1ccccc1)c1ccc2ccccc2c1</smiles>

N,4-dimethyl-N-(1-(naphthalen-2-yl)-4-phenylbutyl)benzenesulfonamide (19) was prepared according to Method $\mathrm{C}$. The following amounts of reagents were used: 4-methyl- $\mathrm{N}-(1-$ (naphthalen-2-yl)-4-phenylbutyl)benzenesulfonamide ( $0.50 \mathrm{~g}, 1.2 \mathrm{mmol}, 1.0$ equiv), $\mathrm{NaH}$ ( $36 \mathrm{mg}, 1.5 \mathrm{mmol}$, 1.3 equiv), methyl iodide ( $80 . \mu \mathrm{L}, 1.3 \mathrm{mmol}, 1.1$ equiv) and THF ( $23 \mathrm{~mL}$ ). The residue was purified by flash column chromatography (0-25\% EtOAc/hexanes) to yield the title compound as a pale yellow solid $(0.42 \mathrm{~g}, 0.95 \mathrm{mmol}, 82 \%$ yield $)$. TLC $\mathrm{R}_{\mathrm{f}}=0.3$ (25\% EtOAc/hexanes); ${ }^{1} \mathrm{H}$ NMR $\left(500 \mathrm{MHz}, \mathrm{CDCl}_{3}\right) \delta 7.85(\mathrm{td}, J=8.1,7.1,4.1 \mathrm{~Hz}, 2 \mathrm{H}), 7.80$ $(\mathrm{d}, J=8.5 \mathrm{~Hz}, 1 \mathrm{H}), 7.75(\mathrm{dt}, J=6.1,3.7 \mathrm{~Hz}, 1 \mathrm{H}), 7.72(\mathrm{~d}, J=8.1 \mathrm{~Hz}, 2 \mathrm{H}), 7.58-7.50(\mathrm{~m}, 3 \mathrm{H})$, 
7.46-7.39 (m, 1H), 7.36-7.31 (m, 2H), 7.26 (t, J = 8.3 Hz, 2H), $7.20(\mathrm{~d}, J=7.3 \mathrm{~Hz}, 2 \mathrm{H}), 5.34(\mathrm{t}$, $J=7.7 \mathrm{~Hz}, 1 \mathrm{H}), 2.72(\mathrm{td}, J=7.5,2.3 \mathrm{~Hz}, 2 \mathrm{H}), 2.68(\mathrm{~s}, 3 \mathrm{H}), 2.44(\mathrm{~s}, 3 \mathrm{H}), 2.16-2.06(\mathrm{~m}, 1 \mathrm{H}), 1.88$ (ddd, $J=15.6,14.0,7.6 \mathrm{~Hz}, 1 \mathrm{H}), 1.70$ (quint, $J=9.0 \mathrm{~Hz}, 2 \mathrm{H}) ;{ }^{13} \mathrm{C} \mathrm{NMR}\left(126 \mathrm{MHz} \mathrm{CDCl}_{3}\right) \delta$ $143.1,141.9,135.9,133.1,132.9,129.9,129.6$ (2C), 128.53 (2C), 128.46 (2C), 128.3, 128.1, 127.7, 127.6, 127.3 (2C), 126.7, 126.4, 126.3, 126.0, 60.0, 35.5, 30.0, 28.9, 28.3, 21.6; HRMS (TOF MS $\mathrm{ES}+) \mathrm{m} / z:[\mathrm{M}+\mathrm{Na}]$ calcd. for $\mathrm{C}_{28} \mathrm{H}_{29} \mathrm{NO}_{2} \mathrm{~S} 466.1817$, found 466.1816 .<smiles>CN([13CH3])C(CC1CCCCC1)c1cc2ccccc2s1</smiles>

$N$-(1-benzo[b]thiophen-2-yl)-2-cyclohexylethyl)-N,4-dimethylbenzenesulfonamide (21) was prepared according to Method C. The following amounts of reagents were used: $N-(1-$ (benzo[b]thiophen-2-yl)-2-cyclohexylethyl)-4-methylbenzenesulfonamide $(170 \mathrm{mg}$, $0.41 \mathrm{mmol}, 1.0$ equiv), $\mathrm{NaH}$ ( $31 \mathrm{mg}, 0.53 \mathrm{mmol}, 1.3$ equiv), methyl iodide (30. $\mu \mathrm{L}, 0.45 \mathrm{mmol}$, 1.1 equiv), and THF ( $8.2 \mathrm{~mL})$. The residue was purified by flash column chromatography (20\% EtOAc/hexanes) to afford the title compound as a clear, colorless oil (170 mg, 0.40 mmol, 98\% yield). TLC $\mathrm{R}_{\mathrm{f}}=0.40$ (10\% EtOAc/hexanes); ${ }^{1} \mathrm{H}$ NMR (500 MHz, CDCl3) $\delta 7.73$ $(\mathrm{d}, J=7.8 \mathrm{~Hz}, 1 \mathrm{H}), 7.70(\mathrm{~d}, J=8.3 \mathrm{~Hz}, 2 \mathrm{H}), 7.66(\mathrm{~d}, J=7.2 \mathrm{~Hz}, 1 \mathrm{H}), 7.30(\mathrm{dtd}, J=16.4,7.2$, $1.3 \mathrm{~Hz}, 2 \mathrm{H}), 7.24(\mathrm{~d}, J=8.0 \mathrm{~Hz}, 2 \mathrm{H}), 7.05(\mathrm{~s}, 1 \mathrm{H}), 5.50(\mathrm{t}, J=7.6,1 \mathrm{H}), 2.71(\mathrm{~s}, 3 \mathrm{H}), 2.39(\mathrm{~s}$, $3 \mathrm{H}), 1.92-1.78(\mathrm{~m}, 2 \mathrm{H}), 1.76-1.59(\mathrm{~m}, 5 \mathrm{H}), 1.33-1.23(\mathrm{~m}, 1 \mathrm{H}), 1.20-1.10(\mathrm{~m}, 3 \mathrm{H}), 1.04-0.83(\mathrm{~m}$, 2H); ${ }^{13} \mathrm{C}$ NMR (125.8 MHz, CDCl3) $\delta$ 144.1, 143.0, 139.4, 139.1, 137.0, 129.4 (2C), 127.2 (2C), $124.2,123.3,122.3,122.5,122.1,53.9,40.3,34.0,33.4,33.0,28.7,26.3,26.0,25.9,21.4$; HRMS (TOF MS ES+) $m / z[\mathrm{M}+\mathrm{Na}]^{+}$calcd. for $\mathrm{C}_{24} \mathrm{H}_{29} \mathrm{NO}_{2} \mathrm{~S}_{2} \mathrm{Na} 450.1537$, found 450.1530 .<smiles>CN(C)C(CC1CCCCC1)c1ccc2ccccc2c1</smiles>

$N$-(2-cyclohexyl-1(napthalen-2-yl)ethyl)-N,4-dimethylbenzenesulfonamide (23) was prepared according to Method $\mathrm{C}$. The following amounts of reagents were used: $\mathrm{N}-(2-$ cyclohexyl-1-(naphthalen-2-yl)ethyl)-4-methylbenzenesulfonamide (190 mg, $0.46 \mathrm{mmol}$, 1.0 equiv), $\mathrm{NaH}$ (17 mg, $0.70 \mathrm{mmol}, 1.5$ equiv), methyl iodide (40. $\mu \mathrm{L}, 0.60 \mathrm{mmol}, 1.1$ equiv), and THF $(11 \mathrm{~mL})$. The residue was purified by flash column chromatography $(0-10 \%$ EtOAc/hexanes) to afford the title compound as a yellow oil (180 mg, $0.43 \mathrm{mmol}, 86 \%$ yield). TLC $\mathrm{R}_{\mathrm{f}}=0.4(10 \% \mathrm{EtOAc} /$ hexanes $) ; 1 \mathrm{H} \mathrm{NMR}(500 \mathrm{MHz}, \mathrm{CDCl} 3) \delta 7.87(\mathrm{~d}, \mathrm{~J}=9.2 \mathrm{~Hz}$, $1 \mathrm{H}), 7.82(\mathrm{dd}, J=11.3,7.5 \mathrm{~Hz}, 2 \mathrm{H}), 7.75(\mathrm{~d}, J=8.2 \mathrm{~Hz}, 2 \mathrm{H}), 7.65$ (br s, 1H), 7.56-7.51 (m, 2H), $7.49(\mathrm{dd}, J=8.5,1.5 \mathrm{~Hz}, 1 \mathrm{H}), 7.30(\mathrm{~d}, J=8.0 \mathrm{~Hz}, 2 \mathrm{H}), 5.45(\mathrm{br} \mathrm{t}, J=7.7 \mathrm{~Hz}, 1 \mathrm{H}), 2.72(\mathrm{~s}, 3 \mathrm{H})$, $2.46(\mathrm{~s}, 3 \mathrm{H}), 2.02-1.88(\mathrm{~m}, 2 \mathrm{H}), 1.81(\mathrm{~d}, J=12.5 \mathrm{~Hz}, 1 \mathrm{H}), 1.78-1.72(\mathrm{~m}, 2 \mathrm{H}), 1.72-1.60(\mathrm{~m}$, 2H), 1.26- 1.12 (m, 4H), 1.09-0.87 (m, 2H); 13C NMR (125.8 MHz, CDCl3) $\delta 143.1,137.6$, 136.3, 133.1, 132.9, 129.6 (2C), 128.2, 128.1, 127.6, 127.3 (2C), 126.8, 126.6, 126.2, 57.5 (2C), $38.2,34.2,33.5,33.4,28.9,26.6,26.22,26.21,21.6$; HRMS (TOF MS ES+) $m / z$ [M + Na]+ calcd. for C26H31NO2SNa 444.1973, found 444.1968 .<smiles>C1=C(c2ccccc2)CC(c2cc3ccccc3s2)[Al]1</smiles>

2-(Benzo[b]thiophen-2-yl)-4-phenyl-1-tosyl-1,2,3,6-tetrahydropyridine (30) was prepared according to Method D. The following amounts of reagents were used: imine 28 (240 mg, $0.75 \mathrm{mmol}, 1.0$ equiv), buta-1,3-dien-2-ylbenzene (190 mg, $1.5 \mathrm{mmol}, 2.0$ equiv) [76], $\mathrm{FeCl}_{3}$ (6.0 $\mathrm{mg}$, 40. $\mu \mathrm{mol}, 5.0 \mathrm{~mol} \%)$, and PhMe (10. mL). The residue was purified by column 
chromatography (0-10\% EtOAc/hexanes) to afford the title compound as a yellow oil (154 mg, $0.34 \mathrm{mmol}, 46 \%$ yield). TLC R $\mathrm{f}_{\mathrm{f}}=0.5$ (20\% EtOAc/hexanes); ${ }^{1} \mathrm{H}$ NMR (500 MHz, $\left.\mathrm{CDCl}_{3}\right) \delta 7.71(\mathrm{~d}, J=8.1 \mathrm{~Hz}, 2 \mathrm{H}), 7.68(\mathrm{~d}, J=7.8 \mathrm{~Hz}, 1 \mathrm{H}), 7.62(\mathrm{~d}, J=7.8 \mathrm{~Hz}, 1 \mathrm{H}), 7.36-$ $7.22(\mathrm{~m}, 8 \mathrm{H}), 7.18(\mathrm{~d}, J=8.0 \mathrm{~Hz}, 2 \mathrm{H}), 7.10(\mathrm{~s}, 1 \mathrm{H}), 5.95(\mathrm{~s}, 1 \mathrm{H}), 5.80(\mathrm{~d}, J=6.1 \mathrm{~Hz}, 1 \mathrm{H}), 4.35$ $(\mathrm{dt}, J=18.6,3.5 \mathrm{~Hz}, 1 \mathrm{H}), 3.84(\mathrm{dq}, J=18.7,2.8 \mathrm{~Hz}, 1 \mathrm{H}), 2.96(\mathrm{ddt}, J=16.3,6.4,3.2 \mathrm{~Hz}, 1 \mathrm{H})$, $2.87(\mathrm{~d}, J=17.2 \mathrm{~Hz}, 1 \mathrm{H}), 2.33(\mathrm{~s}, 3 \mathrm{H}) ;{ }^{13} \mathrm{C}$ NMR $\left(126 \mathrm{MHz} \mathrm{CDCl}_{3}\right) \delta 144.9,144.6,143.6$, $140.0,139.9,129.9$ (2C), 129.2, 128.8 (2C), 128.4, 127.4 (2C), 126.8 (2C), 124.5, 124.3, 123.5, $122.3,122.3,53.9,42.2,37.1,36.7,31.7,21.7$.

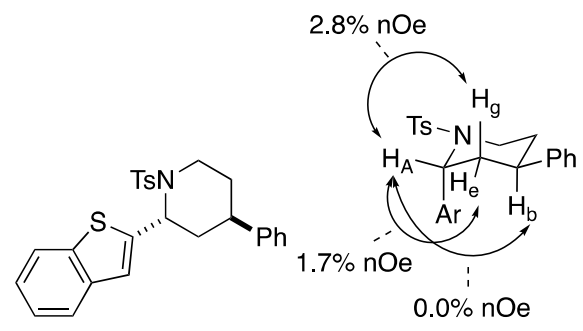

2-(Benzo[b]thiophen-2-yl)-4-phenyl-1-tosylpiperidine (24) was prepared according to Method E. The following amounts of reagents were used: substrate $30(100 \mathrm{mg}, 0.22 \mathrm{mmol}$, 1.0 equiv), $\mathrm{Pd} / \mathrm{C}(20 \mathrm{mg}), \mathrm{DCM}(2.0 \mathrm{~mL})$ and $\mathrm{MeOH}(5.0 \mathrm{~mL})$. The residue was purified by column chromatography $(0-10 \%$ EtOAc/hexanes) to afford the title compound as a pale yellow oil ( $24 \mathrm{mg}, 53 \mu \mathrm{mol}, 25 \%$ yield, $>20: 1 \mathrm{dr}$ trans:cis). The $\mathrm{dr}$ was determined based on the integration of the resonances attributed to the benzylic hydrogens in the ${ }^{1} \mathrm{H}$ NMR spectrum. The relative configuration was assigned based on nOe analysis. TLC $\mathrm{R}_{\mathrm{f}}=0.5\left(20 \%\right.$ EtOAc/hexanes); ${ }^{1} \mathrm{H}$ NMR $\left(500 \mathrm{MHz}, \mathrm{CDCl}_{3}\right) \delta 7.81(\mathrm{~d}, J=8.4 \mathrm{~Hz}, 2 \mathrm{H}), 7.77$ $(\mathrm{d}, J=7.9 \mathrm{~Hz}, 1 \mathrm{H}), 7.68(\mathrm{~d}, J=7.7 \mathrm{~Hz}, 1 \mathrm{H}), 7.40-7.26(\mathrm{~m}, 6 \mathrm{H}), 7.23-7.18(\mathrm{~m}, 1 \mathrm{H}), 7.13(\mathrm{~d}$, $J=1.5 \mathrm{~Hz}, 1 \mathrm{H}), 7.06(\mathrm{~d}, J=7.4 \mathrm{~Hz}, 2 \mathrm{H}), 5.70(\mathrm{~d}, J=5.2 \mathrm{~Hz}, 1 \mathrm{H}), 4.03(\mathrm{~d}, J=14.0 \mathrm{~Hz}, 1 \mathrm{H})$, $3.34(\mathrm{ddd}, J=14.1,12.7,3.0 \mathrm{~Hz}, 1 \mathrm{H}), 2.93(\mathrm{tt}, J=12.6,3.6 \mathrm{~Hz}, 1 \mathrm{H}), 2.43(\mathrm{~s}, 3 \mathrm{H}), 2.33(\mathrm{~d}$, $J=13.0 \mathrm{~Hz}, 1 \mathrm{H}), 2.01(\mathrm{td}, J=13.5,5.5 \mathrm{~Hz}, 1 \mathrm{H}), 1.68(\mathrm{~d}, J=12.5 \mathrm{~Hz}, 1 \mathrm{H}), 1.61(\mathrm{td}, J=12.7$, $4.4 \mathrm{~Hz}, 1 \mathrm{H}) ;{ }^{13} \mathrm{C}$ NMR $\left(151 \mathrm{MHz}, \mathrm{CDCl}_{3}\right) \delta 144.8,144.5,139.9,139.8,129.8(2 \mathrm{C}), 129.1,128.7$ (2C), 128.3, 127.3 (2C), 126.7 (2C), 124.4, 124.2, 123.4, 122.2, 122.2, 76.8, 53.8, 42.1, 36.9, 36.6, 31.6, 21.6; HRMS (TOF MS ES+) $m / z$ [M+Na] calcd. for $\mathrm{C}_{26} \mathrm{H}_{25} \mathrm{NO}_{2} \mathrm{~S}_{2} \mathrm{Na} 470.1224$, found 470.1228 .<smiles></smiles>

4-Methyl-2-(naphthalen-2-yl)-1-tosyl-1,2,3,6-tetrahydropyridine (31) was prepared according to Method D. The following amounts of reagents were used: imine $28(0.31 \mathrm{~g}, 1.0 \mathrm{mmol}$, 1.0 equiv), isoprene ( $1.5 \mathrm{~mL}, 15 \mathrm{mmol}, 15$ equiv), $\mathrm{FeCl}_{3}(16 \mathrm{mg}, 0.10 \mathrm{mmol}, 10 . \mathrm{mol} \%)$, and PhMe (10. $\mathrm{mL}, 0.10 \mathrm{M})$. The residue was purified by flash column chromatography (0-5\% EtOAc/hexanes) to afford the title compound as a yellow oil $(150 \mathrm{mg}, 0.40 \mathrm{mmol}$, $40 \%$ yield). TLC $\mathrm{R}_{\mathrm{f}}=0.5$ (20\% EtOAc/hexanes); ${ }^{1} \mathrm{H}$ NMR (500 MHz, $\left.\mathrm{CDCl}_{3}\right) \delta 7.78-7.71$ $(\mathrm{m}, 3 \mathrm{H}), 7.69(\mathrm{~d}, J=8.3 \mathrm{~Hz}, 2 \mathrm{H}), 7.56(\mathrm{~s}, 1 \mathrm{H}), 7.48-7.40(\mathrm{~m}, 3 \mathrm{H}), 7.20(\mathrm{~d}, J=8.1 \mathrm{~Hz}, 2 \mathrm{H}), 5.43$ $(\mathrm{d}, J=3.5 \mathrm{~Hz}, 1 \mathrm{H}), 5.29(\mathrm{~s}, 1 \mathrm{H}), 4.11(\mathrm{~d}, J=18.0 \mathrm{~Hz}, 1 \mathrm{H}), 3.35(\mathrm{~d}, J=18.1 \mathrm{~Hz}, 1 \mathrm{H}), 2.40-2.30$ $(\mathrm{m}, 2 \mathrm{H}), 2.35(\mathrm{~s}, 3 \mathrm{H}), 1.68(\mathrm{~s}, 3 \mathrm{H})$.

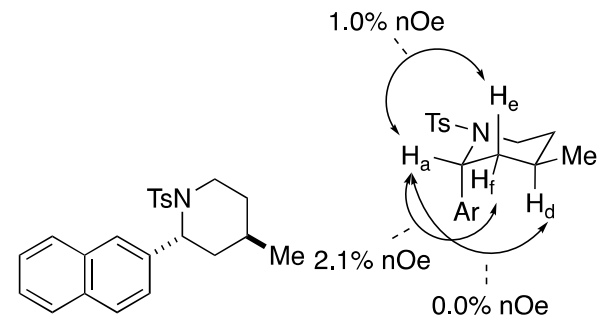


4-Methyl-2-(naphthalen-2-yl)-1-tosylpiperidine (25) was prepared according to Method E. The following amounts of reagents were used: substrate 31 ( $53 \mathrm{mg}, 0.14 \mathrm{mmol}, 1.0$ equiv), $\mathrm{Pd} / \mathrm{C}(27 \mathrm{mg}), \mathrm{DCM}(1.0 \mathrm{~mL})$ and $\mathrm{MeOH}(1.0 \mathrm{~mL})$. The residue was purified by flash column chromatography (0-10\% EtOAc/hexanes) to afford the title compound as a pale yellow oil (9.6 mg, $25 \mu \mathrm{mol}, 18 \%$ yield, 6:1 dr cis:trans). The $\mathrm{dr}$ was determined based on the integration of the resonances attributed to the benzylic hydrogens in the ${ }^{1} \mathrm{H}$ NMR spectrum. The relative configuration was assigned based on nOe analysis. For clarity, the ${ }^{1} \mathrm{H}$ NMR and ${ }^{13} \mathrm{C}$ NMR data of the major and minor diastereomers have been tabulated individually.

TLC $\mathrm{R}_{\mathrm{f}}=0.5$ (10\% EtOAc/hexanes); HRMS (TOF MS ES+) $\mathrm{m} / z$ [M $\left.+\mathrm{H}\right]$ calcd. for $\mathrm{C}_{23} \mathrm{H}_{26} \mathrm{NO}_{2} \mathrm{~S}_{2} 380.1684$, found 380.1689 .

Major Diastereomer: ${ }^{1} \mathrm{H}$ NMR $\left(500 \mathrm{MHz}, \mathrm{CDCl}_{3}\right) \delta 7.80-7.76(\mathrm{~m}, 4 \mathrm{H}), 7.73-7.71(\mathrm{~m}$, $1 \mathrm{H}), 7.63(\mathrm{~s}, 1 \mathrm{H}), 7.46-7.44(\mathrm{~m}, 3 \mathrm{H}), 7.28(\mathrm{~d}, J=8.0 \mathrm{~Hz}, 2 \mathrm{H}), 5.48(\mathrm{~d}, J=4.5 \mathrm{~Hz}, 1 \mathrm{H}), 3.96(\mathrm{~d}$, $J=14.4 \mathrm{~Hz}, 1 \mathrm{H}), 3.06(\mathrm{ddd}, J=14.0,13.2,3.1 \mathrm{~Hz}, 1 \mathrm{H}), 2.69(\mathrm{~d}, J=25.9 \mathrm{~Hz}, 1 \mathrm{H}), 2.42(\mathrm{~s}, 3 \mathrm{H})$, $2.30(\mathrm{~d}, J=13.3 \mathrm{~Hz}, 1 \mathrm{H}), 1.43-1.36(\mathrm{~m}, 2 \mathrm{H}), 0.98(\mathrm{ddd}, J=24.5,12.4,4.5 \mathrm{~Hz}, 1 \mathrm{H}), 0.82(\mathrm{~d}$, $J=6.5 \mathrm{~Hz}, 3 \mathrm{H}) ;{ }^{13} \mathrm{C}$ NMR $\left(151 \mathrm{MHz}, \mathrm{CDCl}_{3}\right) \delta 143.1,138.8,136.7,133.3,132.3,129.7(2 \mathrm{C})$, 128.4, 128.0, 127.5, 127.1 (2C), 126.1, 125.9, 125.8, 125.1, 55.6, 42.0, 36.0, 33.0, 25.3, 22.2, 21.5.

Minor Diastereomer: ${ }^{1} \mathrm{H}$ NMR $\left(500 \mathrm{MHz}, \mathrm{CDCl}_{3}\right) \delta 7.80-7.76(\mathrm{~m}, 4 \mathrm{H}), 7.73-7.71(\mathrm{~m}$, $1 \mathrm{H}), 7.63(\mathrm{~s}, 1 \mathrm{H}), 7.46-7.44(\mathrm{~m}, 3 \mathrm{H}), 7.28(\mathrm{~d}, J=8.0 \mathrm{~Hz}, 2 \mathrm{H}), 5.25(\mathrm{~d}, J=4.9 \mathrm{~Hz}, 1 \mathrm{H}), 3.88(\mathrm{~d}$, $J=10.6 \mathrm{~Hz}, 1 \mathrm{H}), 3.04-2.98(\mathrm{~m}, 1 \mathrm{H}), 2.42(\mathrm{~s}, 3 \mathrm{H}), 2.38(\mathrm{~d}, J=3.86 \mathrm{~Hz}, 1 \mathrm{H}), 2.14(\mathrm{~d}, J=13.7 \mathrm{~Hz}$, 1H), $1.43-1.36(\mathrm{~m}, 2 \mathrm{H}), 0.79(\mathrm{~d}, J=6.5 \mathrm{~Hz}, 3 \mathrm{H}), 0.75(\mathrm{~d}, J=6.4 \mathrm{~Hz}, 1 \mathrm{H}) ;{ }^{13} \mathrm{C}$ NMR $(151 \mathrm{MHz}$, $\left.\mathrm{CDCl}_{3}\right) \delta 143.1,138.8,136.7,133.3,132.3,129.6$ (2C), 129.3, 128.0, 127.6, 127.1 (2C), 126.1, $125.9,125.8,124.0,55.2,41.8,36.0,33.1,25.2,23.3,21.5$.

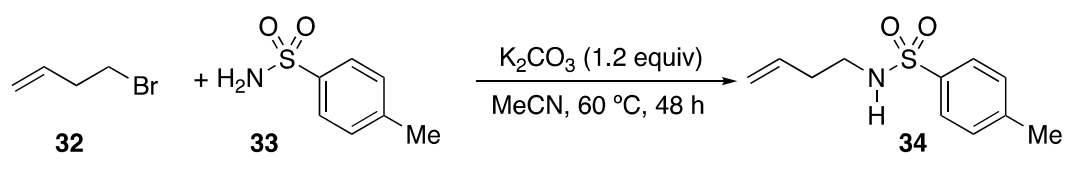

$N$-(but-3-en-1-yl)-4-methylbenzenesulfonamide (34) was prepared according to a procedure reported by Jiang [77]. To a flame-dried flask equipped with a stir bar was added 4bromo-1-butene 32 ( $4.1 \mathrm{~mL}, 40$. mmol, 1.0 equiv), $p$-toluenesulfonamide 33 ( $6.8 \mathrm{~g}, 40 . \mathrm{mmol}$, 1.0 equiv), $\mathrm{K}_{2} \mathrm{CO}_{3}(6.6 \mathrm{~g}, 48 \mathrm{mmol}, 1.2$ equiv), and $\mathrm{MeCN}(160 \mathrm{~mL})$. The mixture was heated to $60^{\circ} \mathrm{C}$ and allowed to stir for $3 \mathrm{~d}$. The reaction mixture was quenched with saturated $\mathrm{NH}_{4} \mathrm{Cl}(100 \mathrm{~mL})$ and extracted with EtOAc $(3 \times 50 \mathrm{~mL})$. The combined organic layers were washed with $\mathrm{H}_{2} \mathrm{O}(50 \mathrm{~mL})$ and brine $(50 \mathrm{~mL})$, dried over $\mathrm{Na}_{2} \mathrm{SO}_{4}$, and concentrated in vacuo. The residue was purified by column chromatography (5-25\% EtOAc/hexanes) to afford the title compound as a clear, colorless oil $(5.4 \mathrm{~g}, 24 \mathrm{mmol}, 60 \%)$. Analytical data are consistent with literature values [77]. ${ }^{1} \mathrm{H}$ NMR: $\left(400 \mathrm{MHz}, \mathrm{CDCl}_{3}\right) \delta 7.76(\mathrm{~d}, J=8.2,2 \mathrm{H})$, $7.30(\mathrm{~d}, J=8.1,2 \mathrm{H}), 5.63(\mathrm{ddt}, J=17.1,10.4,6.8,1 \mathrm{H}), 5.11(\mathrm{br} \mathrm{s}, 1 \mathrm{H}), 5.02-4.93(\mathrm{~m}, 2 \mathrm{H}), 2.99$ $(\mathrm{q}, J=6.7,2 \mathrm{H}), 2.41(\mathrm{~s}, 3 \mathrm{H}), 2.20(\mathrm{q}, J=6.9,2 \mathrm{H})$.<smiles>OC1CCN(c2ccc3ccccc3c2)CC1</smiles>

2-(Naphthalen-2-yl)-1-tosylpiperidin-4-ol (35) was prepared according to Method F. The following amounts of reagents were used: 2-napthaldehyde ( $0.94 \mathrm{~g}, 6.0 \mathrm{mmol}, 1.0$ equiv), homoallylic sulfonamide 35 ( $1.1 \mathrm{~mL}, 6.0 \mathrm{mmol}, 1.0$ equiv), TFA ( $4.6 \mathrm{~mL}, 60 . \mathrm{mmol}, 10$ equiv), and $\mathrm{CH}_{2} \mathrm{Cl}_{2}(60 \mathrm{~mL}, 0.10 \mathrm{M})$. The residue was purified by flash column chromatography $(0-30 \%$ EtOAc/hexanes) to afford the title compound as an orange solid $(0.72 \mathrm{~g}, 1.8 \mathrm{mmol}$, $31 \%$ yield, 5:1 dr trans:cis). The $\mathrm{dr}$ was determined based on the integration of the resonances attributed to the benzylic hydrogens in the ${ }^{1} \mathrm{H}$ NMR spectrum. The relative configuration of the major $\mathbf{3 4}$ was assigned based on analogy to compound 24. For clarity, the ${ }^{1} \mathrm{H}$ NMR data of the major and minor diastereomers have been tabulated individually. 
TLC $R_{\mathrm{f}}=0.1$ (30\% EtOAc/hexanes, stained with CAM); HRMS (TOF MS ES+) $\mathrm{m} / \mathrm{z}$ $[\mathrm{M}+\mathrm{H}]$ calcd. for $\mathrm{C}_{22} \mathrm{H}_{24} \mathrm{NO}_{3} \mathrm{~S} 382.1477$, found 382.1483.

Major Diastereomer: ${ }^{1} \mathrm{H}$ NMR $\left(500 \mathrm{MHz}, \mathrm{CDCl}_{3}\right) \delta 7.79-7.77(\mathrm{~m}, 4 \mathrm{H}), 7.68(\mathrm{~s}, 1 \mathrm{H})$, 7.49-7.41 (m, 4H), $7.29(\mathrm{~d}, J=8.1 \mathrm{~Hz}, 2 \mathrm{H}), 5.54(\mathrm{~d}, J=4.5 \mathrm{~Hz}, 1 \mathrm{H}), 3.99(\mathrm{~d}, J=15.0 \mathrm{~Hz}, 1 \mathrm{H})$, $3.74(\mathrm{tt}, J=10.9,7.9 \mathrm{~Hz}, 1 \mathrm{H}), 3.03(\mathrm{td}, J=15.3,2.7 \mathrm{~Hz}, 1 \mathrm{H}), 2.63(\mathrm{dt}, J=13.3,2.0 \mathrm{~Hz} 1 \mathrm{H}), 2.43$ $(\mathrm{s}, 3 \mathrm{H}), 1.70$ (br s, $1 \mathrm{H}), 1.58$ (ddd $J=13.6,11.3,5.5 \mathrm{~Hz}, 1 \mathrm{H}), 1.26-1.18(\mathrm{~m}, 2 \mathrm{H}) .{ }^{13} \mathrm{C}$ NMR $\left(151 \mathrm{MHz}, \mathrm{CDCl}_{3}\right) \delta 143.5,138.3,135.9,133.3,132.5,130.0$ (2C), 128.7, 128.0, 127.5, 127.0 (2C), 126.3, 126.2, 125.5, 124.7, 64.7, 55.8, 40.7, 36.2, 33.8, 21.6.

Minor Diastereomer: ${ }^{1} \mathrm{H}$ NMR $\left(500 \mathrm{MHz}, \mathrm{CDCl}_{3}\right) \delta 7.74-7.70(\mathrm{~m}, 4 \mathrm{H}), 7.63(\mathrm{~s}, 1 \mathrm{H})$, $7.60(\mathrm{~d}, J=8.3 \mathrm{~Hz}, 2 \mathrm{H}), 7.49-7.41(\mathrm{~m}, 2 \mathrm{H}), 7.16(\mathrm{~d}, J=8.4 \mathrm{~Hz}, 2 \mathrm{H}), 5.10(\mathrm{t}, J=5.1 \mathrm{~Hz}, 1 \mathrm{H})$, $3.99(\mathrm{~d}, J=15.0 \mathrm{~Hz}, 1 \mathrm{H}), 3.67(\mathrm{tt}, J=13.4,4.6 \mathrm{~Hz}, 1 \mathrm{H}), 3.03(\mathrm{td}, J=15.3,2.7 \mathrm{~Hz}, 1 \mathrm{H}), 2.63(\mathrm{dt}$, $J=13.3,2.0 \mathrm{~Hz} 1 \mathrm{H}), 2.36(\mathrm{~s}, 3 \mathrm{H}), 1.81-1.73(\mathrm{~m}, 1 \mathrm{H}), 1.67(\mathrm{br} \mathrm{s}, 1 \mathrm{H}), 1.26-1.18(\mathrm{~m}, 2 \mathrm{H}) .{ }^{13} \mathrm{C}$ NMR (151 MHz, CDCl $)$ ) 143.3, 137.7, 135.9, 133.2, 132.5, 129.6 (2C), 128.7, 128.2, 127.5, 127.2 (2C), 126.3, 126.0, 125.3, 124.8, 65.1, 55.5, 39.0, 37.0, 31.9, 21.5 .

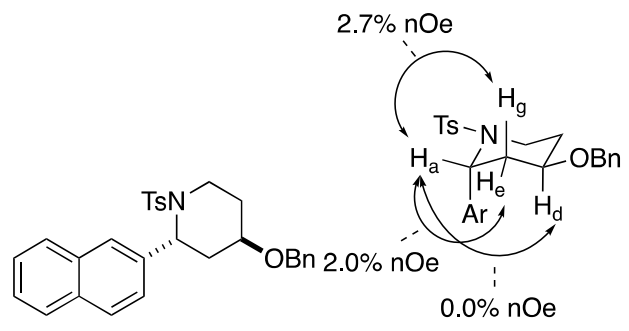

4-(Benzyloxy)-2-(naphthalen-2-yl)-1-tosylpiperidine (26) was prepared according to Method G. The following amounts of reagents were used: alcohol $35(0.25 \mathrm{~g}, 0.66 \mathrm{mmol}, 1.0$ equiv), $\mathrm{NaH}$ (63 mg, $2.6 \mathrm{mmol}$. 4.0 equiv), benzyl bromide (90. $\mu \mathrm{L}, 0.73 \mathrm{mmol}, 1.1$ equiv), and THF $(2.3 \mathrm{~mL}, 0.2 \mathrm{M})$. The residue was purified by column chromatography $(0-10 \%$ EtOAc/hexanes) to afford the title compound as a white solid $(140 \mathrm{mg}, 0.30 \mathrm{mmol}, 56 \%$ yield, 5:1 dr trans:cis). The $\mathrm{dr}$ was determined based on the integration of the resonances attributed to the benzylic hydrogens in the ${ }^{1} \mathrm{H}$ NMR spectrum. The relative configuration was assigned based on nOe analysis. For clarity, the ${ }^{1} \mathrm{H}$ NMR and ${ }^{13} \mathrm{C}$ NMR data of the major and minor diastereomers have been tabulated individually.

TLC $R_{\mathrm{f}}=0.8$ (20\% EtOAc/hexanes, stained with CAM); HRMS (TOF MS ES+) $\mathrm{m} / \mathrm{z}$ $[\mathrm{M}+\mathrm{Na}]$ calcd. for $\mathrm{C}_{29} \mathrm{H}_{29} \mathrm{NO}_{3} \mathrm{SNa} 494.1766$, found 494.1758 .

Major Diastereomer: ${ }^{1} \mathrm{H}$ NMR $\left(500 \mathrm{MHz}, \mathrm{CDCl}_{3}\right) \delta 7.80(\mathrm{~d}, J=8.1 \mathrm{~Hz}, 4 \mathrm{H}), 7.70(\mathrm{~d}$, $1 \mathrm{H}), 7.61(\mathrm{~s}, 1 \mathrm{H}), 7.48-7.45(\mathrm{~m}, 3 \mathrm{H}), 7.32-7.26(\mathrm{~m}, 7 \mathrm{H}), 5.55(\mathrm{~d}, J=3.8 \mathrm{~Hz}, 1 \mathrm{H}), 4.50(\mathrm{~d}$, $J=11.9 \mathrm{~Hz}, 1 \mathrm{H}), 4.43(\mathrm{~d}, J=11.9 \mathrm{~Hz}, 1 \mathrm{H}), 4.02(\mathrm{~d}, J=14.7 \mathrm{~Hz}, 1 \mathrm{H}), 3.52(\mathrm{tt}, J=10.8 \mathrm{~Hz}, 1 \mathrm{H})$, $3.04(\mathrm{td}, J=14.5,2.5 \mathrm{~Hz}, 1 \mathrm{H}), 2.68(\mathrm{~d}, J=13.6 \mathrm{~Hz}, 1 \mathrm{H}), 2.43(\mathrm{~s}, 3 \mathrm{H}), 1.80(\mathrm{~d}, J=11.5 \mathrm{~Hz}, 1 \mathrm{H})$, $1.62(\mathrm{ddd}, J=17.7,11.9,6.1 \mathrm{~Hz}, 1 \mathrm{H}), 1.31-1.34(\mathrm{~m}, 1 \mathrm{H}) ;{ }^{13} \mathrm{C} \mathrm{NMR}\left(151 \mathrm{MHz}, \mathrm{CDCl}_{3}\right) \delta 143.4$, $138.3,136.0,133.2$, 132.5, 130.0 (2C), 129.5, 128.6, 128.5 (2C), 128.0, 127.8, 127.7 (2C), 127.5, 127.0 (2C), 126.2, 126.1, 125.5, 124.8, 71.3, 70.2, 55.8, 40.8, 33.4, 30.8, 21.6.

Minor Diastereomer: ${ }^{1} \mathrm{H}$ NMR $\left(500 \mathrm{MHz}, \mathrm{CDCl}_{3}\right) \delta 7.80(\mathrm{~d}, J=8.1 \mathrm{~Hz}, 4 \mathrm{H}), 7.70$ $(\mathrm{d}, 1 \mathrm{H}), 7.59(\mathrm{~s}, 1 \mathrm{H}), 7.48-7.45(\mathrm{~m}, 3 \mathrm{H}), 7.32-7.26(\mathrm{~m}, 2 \mathrm{H}), 7.15(\mathrm{~d}, J=8.2 \mathrm{~Hz}, 1 \mathrm{H}), 7.07(\mathrm{t}$, $J=7.6 \mathrm{~Hz}, 1 \mathrm{H}), 6.96(\mathrm{t}, J=7.3 \mathrm{~Hz}, 2 \mathrm{H}), 6.69(\mathrm{~d}, J=7.6 \mathrm{~Hz}, 1 \mathrm{H}), 5.17(\mathrm{t}, J=5.1 \mathrm{~Hz}, 1 \mathrm{H})$, $4.24,4.20\left(\mathrm{ABq}, J_{\mathrm{AB}}=12.3 \mathrm{~Hz}, 2 \mathrm{H}\right), 3.80-3.68(\mathrm{~m}, 3 \mathrm{H}), 2.53(\mathrm{dt}, J=14.4,9.4 \mathrm{~Hz}, 1 \mathrm{H}), 2.35(\mathrm{~s}$, $3 \mathrm{H}), 2.06(\mathrm{ddd}, J=14.3,5.3,2.9 \mathrm{~Hz}, 1 \mathrm{H}), 1.81-1.80(\mathrm{~m}, 2 \mathrm{H}), 1.31-1.34(\mathrm{~m}, 1 \mathrm{H}) ;{ }^{13} \mathrm{C}$ NMR $\left(151 \mathrm{MHz}, \mathrm{CDCl}_{3}\right) \delta 143.4,138.4,136.0,133.2,132.5,130.0$ (2C), 129.5, 128.6, 128.5 (2C), 128.0, 127.8, 127.7 (2C), 127.5, 127.19 (2C), 127.16, 125.9, 125.6, 125.2, 71.2, 69.8, 55.8, 39.3, $34.2,30.8,21.6$. 


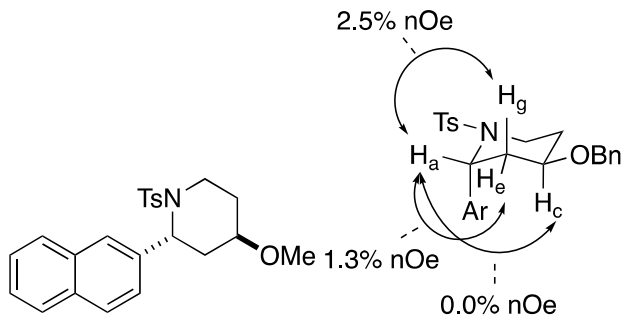

4-Methoxy-2-(naphthalen-2-yl)-1-tosylpiperidine (12) was prepared according to Method G. The following amounts of reagents were used: alcohol 35 (110 mg, $0.30 \mathrm{mmol}, 1.0$ equiv), $\mathrm{NaH}$ (16 mg, $0.67 \mathrm{mmol}, 2.2$ equiv), methyl iodide (20. $\mu \mathrm{L}, 0.33 \mathrm{mmol}, 1.1$ equiv), and THF $(1.5 \mathrm{~mL}, 0.20 \mathrm{M})$. The residue was purified by column chromatography (0-20\% EtOAc/hexanes) to afford the title compound as a pale yellow solid ( $61 \mathrm{mg}, 0.15 \mathrm{mmol}, 52 \%$ yield, 5:1 dr trans:cis). The dr was determined based on the integration of the resonances attributed to the benzylic hydrogens in the ${ }^{1} \mathrm{H}$ NMR spectrum. The relative configuration was assigned based on nOe analysis. For clarity, the ${ }^{1} \mathrm{H}$ NMR and ${ }^{13} \mathrm{C}$ NMR data of the major and minor diastereomers have been tabulated individually.

TLC $\mathrm{R}_{\mathrm{f}}=0.6$ (30\% EtOAc/hexanes, stained with CAM); HRMS (TOF MS ES+) $\mathrm{m} / \mathrm{z}$ $[\mathrm{M}+\mathrm{Na}]$ calcd. for $\mathrm{C}_{23} \mathrm{H}_{25} \mathrm{NO}_{3} \mathrm{SNa} 396.1633$, found 396.1636.

Major Diastereomer: ${ }^{1} \mathrm{H}$ NMR $\left(500 \mathrm{MHz} \mathrm{CDCl}_{3}\right) \delta 7.83-7.76(\mathrm{~m}, 5 \mathrm{H}), 7.72(\mathrm{~s}, 1 \mathrm{H})$, 7.54-7.51 (m, 1H), 7.48-7.46 (m, 2H), $7.29(\mathrm{~d}, J=7.9 \mathrm{~Hz}, 2 \mathrm{H}), 5.56(\mathrm{~s}, 1 \mathrm{H}), 4.04(\mathrm{~d}, J=14.1 \mathrm{~Hz}$, 1H), 3.29 (tt, J=7.4, 3.0 Hz, 1H), $3.25(\mathrm{~s}, 3 \mathrm{H}), 3.09$ (t, $J=13.1 \mathrm{~Hz}, 1 \mathrm{H}), 2.68(\mathrm{~d}, J=13.2 \mathrm{~Hz}$, $1 \mathrm{H}), 2.42(\mathrm{~s}, 3 \mathrm{H}), 1.80(\mathrm{~d}, J=11.5 \mathrm{~Hz}, 1 \mathrm{H}), 1.53(\mathrm{td}, J=12.2 \mathrm{~Hz}, 1 \mathrm{H}), 1.16(\mathrm{qd}, J=11.6,5.8 \mathrm{~Hz}$, $1 \mathrm{H}) ;{ }^{13} \mathrm{C} \mathrm{NMR}\left(151 \mathrm{MHz}, \mathrm{CDCl}_{3}\right) \delta 143.4,138.4,136.1,133.4,132.5,130.0$ (2C), 126.7, 128.1, 127.6, 127.0 (2C), 126.3, 126.1, 125.5, 124.7, 73.2, 55.8, 55.5, 40.8, 33.2, 30.2, 21.6.

Minor Diastereomer: ${ }^{1} \mathrm{H}$ NMR $\left(500 \mathrm{MHz}_{\mathrm{CDCl}}\right) \delta 7.83-7.76(\mathrm{~m}, 5 \mathrm{H}), 7.72(\mathrm{~s}, 1 \mathrm{H})$, $7.54-7.51(\mathrm{~m}, 1 \mathrm{H}), 7.48-7.46(\mathrm{~m}, 2 \mathrm{H}), 7.29(\mathrm{~d}, J=7.9 \mathrm{~Hz}, 2 \mathrm{H}), 4.99(\mathrm{t}, J=5.38 \mathrm{~Hz}, 1 \mathrm{H})$, 3.77-3.71 (m, 1H), $3.60(\mathrm{dt}, J=13.6,4.6 \mathrm{~Hz}, 1 \mathrm{H}), 3.42(\mathrm{br} \mathrm{s}, 1 \mathrm{H}), 3.03(\mathrm{~s}, 3 \mathrm{H}), 2.37-2.29(\mathrm{~m}$, $1 \mathrm{H}), 2.33(\mathrm{~s}, 3 \mathrm{H}), 2.02(\mathrm{~d}, J=15.2 \mathrm{~Hz}, 1 \mathrm{H}), 1.86-1.82(\mathrm{~m}, 1 \mathrm{H}), 1.72-1.68(\mathrm{~m}, 1 \mathrm{H}){ }^{13} \mathrm{C} \mathrm{NMR}$ $\left(151 \mathrm{MHz} \mathrm{CDCl}_{3}\right) \delta 143.0,138.3,137.4,133.1,132.5,129.4$ (2C), 126.7, 128.0, 127.5, 127.2 (2C), $125.8,125.7,125.5,125.2,73.5,56.4,55.5,40.0,34.1,29.6,21.5$.

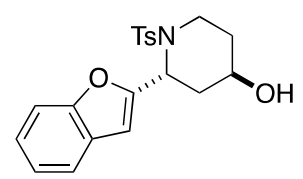

2-(Naphthalen-2-yl)-1-tosylpiperidin-4-ol (36) was prepared according to Method F. The following amounts of reagents were used: 2-benzofurancarboxaldehyde $(0.60 \mathrm{~mL}, 5.0 \mathrm{mmol}$, 1.0 equiv), homoallylic sulfonamide 34 (0.91 mL, $5.0 \mathrm{mmol}, 1.0$ equiv), TFA (3.8 mL, 50 . mmol, 10 equiv), $\mathrm{CH}_{2} \mathrm{Cl}_{2}(50 \mathrm{~mL}, 0.10 \mathrm{M})$. The residue was purified by flash column chromatography (0-50\% EtOAc/hexanes) to afford the title compound as an orange solid (0.42 g, $1.1 \mathrm{mmol}, 22 \%$ yield, 5:1 dr trans:cis). The $\mathrm{dr}$ was determined based on the integration of the resonances attributed to the benzylic hydrogens in the ${ }^{1} \mathrm{H}$ NMR spectrum. The relative configuration was assigned based on analogy to compound 26. For clarity, the ${ }^{1} \mathrm{H}$ NMR data of the major and minor diastereomers have been tabulated individually.

TLC $\mathrm{R}_{\mathrm{f}}=0.1$ (30\% EtOAc/hexanes, stained with CAM).

Major Diastereomer: ${ }^{1} \mathrm{H}$ NMR $\left(500 \mathrm{MHz} \mathrm{CDCl}_{3}\right) \delta 7.65(\mathrm{~d}, J=8.29 \mathrm{~Hz}, 2 \mathrm{H}), 7.46(\mathrm{dd}$, $J=6.75,2.12 \mathrm{~Hz}, 1 \mathrm{H}), 7.21-7.18(\mathrm{~m}, 3 \mathrm{H}), 7.15(\mathrm{~d}, J=8.61 \mathrm{~Hz}, 2 \mathrm{H}), 6.49(\mathrm{t}, J=2.0 \mathrm{~Hz}, 1 \mathrm{H})$, $5.51(\mathrm{~d}, J=5.48 \mathrm{~Hz}, 1 \mathrm{H}), 3.97-3.90(\mathrm{~m}, 2 \mathrm{H}), 3.23(\mathrm{td}, J=13.5,2.7 \mathrm{~Hz}, 1 \mathrm{H}), 2.51-2.45(\mathrm{~m}, 1 \mathrm{H})$, $2.33(\mathrm{~s}, 3 \mathrm{H}), 1.94-1.88(\mathrm{~m}, 1 \mathrm{H}), 1.75(\mathrm{ddd}, J=13.0,11.6,5.9 \mathrm{~Hz}, 1 \mathrm{H}), 1.53(\mathrm{~d}, J=5.0 \mathrm{~Hz}, 1 \mathrm{H})$, $1.44(\mathrm{ddd}, J=24.1,12.8,4.5 \mathrm{~Hz}, 1 \mathrm{H})$.

Minor Diastereomer: ${ }^{1} \mathrm{H}$ NMR $\left(500 \mathrm{MHz}, \mathrm{CDCl}_{3}\right) \delta 7.65(\mathrm{~d}, J=8.29 \mathrm{~Hz}, 2 \mathrm{H}), 7.46(\mathrm{dd}$, $J=6.75,2.12 \mathrm{~Hz}, 1 \mathrm{H}), 7.21-7.18(\mathrm{~m}, 3 \mathrm{H}), 7.15(\mathrm{~d}, J=8.61 \mathrm{~Hz}, 2 \mathrm{H}), 6.51(\mathrm{t}, J=1.1 \mathrm{~Hz}, 1 \mathrm{H})$, 
$5.33(\mathrm{~d}, J=5.6 \mathrm{~Hz}, 1 \mathrm{H}), 4.14-4.10(\mathrm{~m}, 2 \mathrm{H}), 3.64(\mathrm{td}, J=10.9,4.0 \mathrm{~Hz}, 1 \mathrm{H}), 2.51-2.45(\mathrm{~m}, 1 \mathrm{H})$, $2.32(\mathrm{~s}, 3 \mathrm{H}), 2.12(\mathrm{ddd}, J=14.4,6.7,3.3 \mathrm{~Hz}, 1 \mathrm{H}), 1.94-1.88(\mathrm{~m}, 1 \mathrm{H}), 1.53(\mathrm{~d}, J=5.0 \mathrm{~Hz}, 1 \mathrm{H})$, $1.44(\mathrm{ddd}, J=24.1,12.8,4.5 \mathrm{~Hz}, 1 \mathrm{H})$.

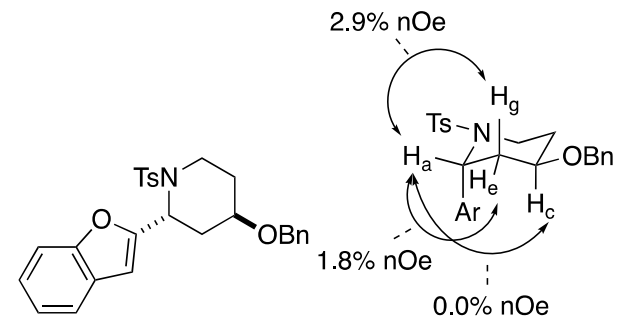

2-(benzofuran-2-yl)-4-(benzyloxy)-1-tosylpiperidine (27) was prepared according to method G. The following amounts of reagents were used: alcohol 36 ( $0.15 \mathrm{~g}, 0.40 \mathrm{mmol}, 1.0 \mathrm{equiv})$, $\mathrm{NaH}$ (46 mg, $1.9 \mathrm{mmol}, 4.7$ equiv), benzyl bromide (52 $\mu \mathrm{L}, 0.44 \mathrm{mmol}, 1.1$ equiv), and THF (3.0 mL, $0.2 \mathrm{M})$. The residue was purified by column chromatography (0-10\% EtOAc/ hexanes) to afford the title compound as a yellow solid $(87 \mathrm{mg}, 0.19 \mathrm{mmol}, 47 \%$ yield, 5:1 dr trans:cis). The dr was determined based on the integration of the resonances attributed to the benzylic hydrogens in the ${ }^{1} \mathrm{H}$ NMR spectrum. The relative configuration was assigned based on nOe analysis. For clarity, the ${ }^{1} \mathrm{H}$ NMR and ${ }^{13} \mathrm{C}$ NMR data of the major and minor diastereomers have been tabulated individually.

TLC $R_{\mathrm{f}}=0.8$ (30\% EtOAc/hexanes, stained with CAM); HRMS (TOF MS ES+) $\mathrm{m} / z$ $[\mathrm{M}+\mathrm{Na}]$ calcd. for $\mathrm{C}_{27} \mathrm{H}_{27} \mathrm{NO}_{4} \mathrm{SNa} 484.1559$, found 484.1542 .

Major Diastereomer: ${ }^{1} \mathrm{H}$ NMR $\left(500 \mathrm{MHz}, \mathrm{CDCl}_{3}\right) \delta 7.65(\mathrm{~d}, J=8.3 \mathrm{~Hz}, 2 \mathrm{H}), 7.45,(\mathrm{~d}$, $J=8.3 \mathrm{~Hz}, 1 \mathrm{H}), 7.30-7.17(\mathrm{~m}, 8 \mathrm{H}), 7.15(\mathrm{~d}, J=8.2 \mathrm{~Hz}, 2 \mathrm{H}), 6.44(\mathrm{~s}, 1 \mathrm{H}), 5.52(\mathrm{~d}, J=5.3 \mathrm{~Hz}$, $1 \mathrm{H}), 4.49(\mathrm{~s}, 2 \mathrm{H}), 3.94(\mathrm{~d}, J=13.7 \mathrm{~Hz}, 1 \mathrm{H}), 3.66(\mathrm{tt}, J=11.2,4.0 \mathrm{~Hz}, 1 \mathrm{H}), 3.20(\mathrm{td}, J=13.4$, $2.6 \mathrm{~Hz}, 1 \mathrm{H}), 2.57(\mathrm{dt}, J=13.2,1.8 \mathrm{~Hz}, 1 \mathrm{H}), 2.32(\mathrm{~s}, 3 \mathrm{H}), 1.97(\mathrm{~d}, J=12.3 \mathrm{~Hz}, 1 \mathrm{H}), 1.78(\mathrm{ddd}$, $J=13.0,11.7,5.8 \mathrm{~Hz}, 1 \mathrm{H}), 1.45(\mathrm{qd}, J=12.8,4.8 \mathrm{~Hz}, 1 \mathrm{H}) ;{ }^{13} \mathrm{C} \mathrm{NMR}\left(151 \mathrm{MHz}, \mathrm{CDCl}_{3}\right) \delta$ $155.3,154.7,143.3,138.2,137.2,129.5$ (2C), 128.5 (2C), 128.1, 128.0, 127.8, 127.1, 126.9, 124.1, $122.9,120.9,111.1,104.8,71.8,70.2,51.7,41.4,34.2,31.3,21.5$.

Minor Diastereomer: ${ }^{1} \mathrm{H}$ NMR $\left(500 \mathrm{MHz} \mathrm{CDCl}_{3}\right) \delta 7.68(\mathrm{~d}, J=8.3 \mathrm{~Hz}, 2 \mathrm{H}), 7.35$, $(\mathrm{d}, J=4.4 \mathrm{~Hz}, 1 \mathrm{H}), 7.30-7.17(\mathrm{~m}, 5 \mathrm{H}), 7.07(\mathrm{t}, J=7.5 \mathrm{~Hz}, 1 \mathrm{H}), 6.99(\mathrm{t}, J=7.6,2 \mathrm{H}), 6.79(\mathrm{~d}$, $J=7.6 \mathrm{~Hz}, 2 \mathrm{H}), 6.42(\mathrm{~s}, 1 \mathrm{H}), 5.36(\mathrm{~d}, J=6.4 \mathrm{~Hz}, 1 \mathrm{H}), 4.28(\mathrm{~s}, 2 \mathrm{H}), 3.76-3.73(\mathrm{~m}, 2 \mathrm{H}), 3.66(\mathrm{tt}$, $J=11.2,4.0 \mathrm{~Hz}, 1 \mathrm{H}), 2.70(\mathrm{~d}, J=14.1 \mathrm{~Hz}, 1 \mathrm{H}), 2.32(\mathrm{~s}, 3 \mathrm{H}), 1.97(\mathrm{~d}, J=12.3 \mathrm{~Hz}, 1 \mathrm{H}), 1.83(\mathrm{~d}$, $J=13.8 \mathrm{~Hz}, 1 \mathrm{H}), 1.72-1.68(\mathrm{~m}, 1 \mathrm{H}) ;{ }^{13} \mathrm{C} \mathrm{NMR}\left(151 \mathrm{MHz}, \mathrm{CDCl}_{3}\right) \delta 155.3,154.7,143.3,138.2$, $137.2,129.5$ (2C), 128.5 (2C), 128.1, 128.0, 127.8, 127.1, 126.9, 123.6, 122.6, 120.7, 110.9, 103.2, $71.8,70.0,49.6,37.6,31.0,29.3,15.3$.

\section{Conclusions}

In conclusion, we have developed a Kumada XC reaction of benzylic sulfonamides with Grignard reagents including methylmagnesium iodide and arylmagnesium iodide. This reaction utilizes readily available starting materials that are not activated prior to the $\mathrm{XC}$ reaction. We have demonstrated that increasing the steric bulk adjacent to the reactive center destabilizes the conformation necessary for $\beta$-hydride elimination to occur. A stereospecific ring opening Kumada XC reaction has been established to synthesize highly substituted acyclic fragments. This work provides a basis for the $\mathrm{XC}$ reaction of simple benzylic sulfonamides.

Supplementary Materials: The following are available online, ${ }^{1} \mathrm{H},{ }^{13} \mathrm{C}, \mathrm{COSY}$ and NOE NMR data are available online.

Author Contributions: K.A.H., C.A.H. and A.C.M. performed the experiments and analyzed the NMR data. K.A.H. wrote the first draft of the paper. E.R.J. conceived, wrote and finalized the paper. All authors have read and agreed to the published version of the manuscript.

Funding: This work was supported by the National Science Foundation (NSF CHE-1900340). 
Institutional Review Board Statement: Not application.

Informed Consent Statement: Not application.

Data Availability Statement: Data sharing not applicable.

Acknowledgments: We gratefully acknowledge Felix Grun and the UC Irvine Mass Spectrometry Facility for mass spectrometry data.

Conflicts of Interest: The authors declare no conflict of interest.

Sample Availability: Samples of the compounds are not available from the authors.

\section{References and Note}

1. De Meijere, A.; Diederich, F. (Eds.) Metal-Catalyzed Cross-Coupling Reactions, 2nd ed.; Wiley-VCH: Winheim, Germany, 2004.

2. Hartwig, J.F. Organotransition Metal Chemistry: From Bonding to Catalysis; University Science Books: Sausalito, CA, USA, 2010.

3. Jana, R.; Pathak, T.P.; Sigman, M.S. Advances in Transition Metal (Pd,Ni,Fe)-Catalyzed Cross-Coupling Reactions Using AlkylOrganometallics as Reaction Partners. Chem. Rev. 2011, 111, 1417-1492. [CrossRef] [PubMed]

4. Cherney, A.H.; Kadunce, N.T.; Reisman, S.E. Enantioselective and Enantiospecific Transition-Metal-Catalyzed Cross-Coupling Reactions of Organometallic Reagents to Construct C-C bonds. Chem. Rev. 2015, 115, 9587-9652. [CrossRef] [PubMed]

5. Choi, J.; Fu, G.C. Transition Metal-Catalyzed Alkyl-Alkyl Bond Formation: Another Dimension in Cross-Coupling Chemistry. Science 2017, 356, eaaf7230. [CrossRef] [PubMed]

6. Campeau, L.-C.; Hazari, N. Cross-Coupling and Related Reactions: Connecting Past Successes to the Development of New Reactions for the Future. Organometallics 2019, 38, 3-35. [CrossRef] [PubMed]

7. Tamaru, Y. (Ed.) Modern Organonickel Chemistry; Wiley-VCH: Weinheim, Germany, 2005.

8. Tasker, S.Z.; Standley, E.A.; Jamison, T.F. Recent Advances in Homogeneous Nickel Catalysis. Nature 2014, 509, $299-309$. [CrossRef] [PubMed]

9. Ogoshi, S. (Ed.) Nickel Catalysis in Organic Synthesis: Methods and Reactions; Wiley: Hoboken, NJ, USA, 2020.

10. Singer, R.A.; Monfette, S.; Bernhardson, D.; Tcyrulnikov, S.; Hubbell, A.K.; Hansen, E.C. Recent Advances in Nonprecious Metal Catalysis. Org. Process. Res. Dev. 2021, 25, 1802-1815. [CrossRef]

11. Rosen, B.M.; Quasdorf, K.W.; Wilson, D.A.; Zhang, N.; Resmerita, A.-M.; Garg, N.K.; Percec, V. Nickel-Catalyzed Cross-Couplings Involving Carbon-Oxygen Bonds. Chem. Rev. 2011, 111, 1346-1416. [CrossRef]

12. Tollefson, E.J.; Hanna, L.E.; Jarvo, E.R. Stereospecific Nickel-Catalyzed Cross-Coupling Reactions of Benzylic Ethers and Esters. Acc. Chem. Res. 2015, 48, 2344-2353. [CrossRef]

13. Yu, D.-G.; Li, B.-J.; Shi, Z.-J. Exploration of New C-O Electrophiles in Cross-Coupling Reactions. Acc. Chem. Res. 2010, 43, 1486-1495. [CrossRef]

14. Ouyang, K.; Hao, W.; Zhang, W.-X.; Xi, Z. Transition-Metal-Catalyzed Cleavage of C-N Single Bonds. Chem. Rev. 2015, 115, 12045-12090. [CrossRef]

15. Wang, W.; Su, Y.; Li, L.; Huang, H. Transition-Metal Catalysed C-N Bond Activation. Chem. Soc. Rev. 2016, 45, 1257-1272. [CrossRef]

16. Pound, S.M.; Watson, M.P. Asymmetric Synthesis via Stereospecific C-N and C-O Bond Activation of Alkyl Amine and Alcohol Derivatives. Chem. Commun. 2018, 54, 12286-12301. [CrossRef] [PubMed]

17. Dander, J.E.; Garg, N.K. Breaking Amides using Nickel Catalysis. ACS Catal. 2017, 7, 1413-1423. [CrossRef] [PubMed]

18. Li, G.; Ma, S.; Szostak, M. Amide Bond Activation: The Power of Resonance. Trends Chem. 2020, 2, 914-928. [CrossRef]

19. Li, M.-B.; Tang, X.-L.; Tian, S.-K. Cross-Coupling of Grignard Reagents with Sulfonyl-Activated sp ${ }^{3}$ Carbon-Nitrogen Bonds. Adv. Synth. Catal. 2011, 353, 1980-1984. [CrossRef]

20. Huang, C.-Y.; Doyle, A.G. The Chemistry of Transition Metals with Three-Membered Ring Heterocycles. Chem. Rev. 2014, 114, 8153-8198. [CrossRef]

21. Lin, B.L.; Clough, C.R.; Hillhouse, G.L. Interactions of Aziridines with Nickel Complexes: Oxidative-Addition and ReductiveElimination Reactions that Break and Make C-N Bonds. J. Am. Chem. Soc. 2002, 124, 2890-2891. [CrossRef]

22. Huang, C.-Y.; Doyle, A.G. Nickel-Catalyzed Negishi Alkylations of Styrenyl Aziridines. J. Am. Chem. Soc. 2012, 134, 9541-9544. [CrossRef]

23. Huang, C.-Y.; Doyle, A.G. Electron-Deficient Olefin Ligands Enable Generation of Quaternary Carbons by Ni-Catalyzed Cross Coupling. J. Am. Chem. Soc. 2015, 137, 5638-5641. [CrossRef]

24. Nielsen, D.K.; Huang, C.-Y.; Doyle, A.G. Directed Nickel-Catalyzed Negishi Cross Coupling of Alkyl Aziridines. J. Am. Chem. Soc. 2013, 135, 13605-13609. [CrossRef]

25. Jensen, K.L.; Standley, E.A.; Jamison, T.F. Highly Regioselective Nickel-Catalyzed Cross-Coupling of N-Tosylaziridines and Alkylzinc Reagents. J. Am. Chem. Soc. 2014, 136, 11145-11152. [CrossRef] [PubMed]

26. Wenkert, E.; Han, A.-L.; Jenny, C.-J. Nickel-Induced Conversion of Carbon-Nitrogen into Carbon-Carbon Bonds. One-Step Transformations of Aryl, Quaternary Ammonium Salts into Alkylarenes and Biaryls. J. Chem. Soc. Chem. Commun. 1988, 975-976. [CrossRef] 
27. Blakey, S.B.; MacMillan, D.W.C. The First Suzuki Cross-Couplings of Aryltrimethylammonium Salts. J. Am. Chem. Soc. 2003, 125, 6046-6047. [CrossRef] [PubMed]

28. Maity, P.; Shacklady-McAtee, D.M.; Yap, G.P.A.; Sirianni, E.R.; Watson, M.P. Nickel-Catalyzed Cross-Couplings of Benzylic Ammonium Salts and Boronic Acids: Stereospecific Formation of Diarylethanes via C-N Bond Activation. J. Am. Chem. Soc. 2013, 135, 280-285. [CrossRef] [PubMed]

29. Shacklady-McAtee, D.M.; Roberts, K.M.; Basch, C.H.; Song, Y.-G.; Watson, M.P. A General, Simple Catalyst for Enantiospecific Cross Couplings of Benzylic Ammonium Triflates and Boronic Acids: No Phosphine Ligand Required. Tetrahedron 2014, 70, 4257-4263. [CrossRef]

30. He, F.; Wang, Z.-X. Nickel-Catalyzed Cross-Coupling of Aryl or -2-Menaphthyl Quaternary Ammonium Triflates with Organoaluminum Reagents. Tetrahedron 2017, 73, 4450-4457. [CrossRef]

31. Bapat, J.B.; Blade, R.J.; Boulton, A.J.; Epsztajn, J.; Katritzky, A.R.; Lewis, J.; Molina-Buendia, P.; Nie, P.-L.; Ramsden, C.A. Pyridines as Leaving Groups in Synthetic Transformations: Nucleophilic Displacements of Amino Groups, and Novel Preparations of Nitriles and Isocyanate. Tetrahedron Lett. 1976, 17, 2691-2694. [CrossRef]

32. Katritzky, A.R.; De Ville, G.; Patel, R.C. Carbon-Alkylation of Simple Nitronate Anions by N-Substituted Pyridiniums. Tetrahedron 1981, 37, 25-30. [CrossRef]

33. Katritzky, A.R.; Marson, C.M. Pyrylium Mediated Transformations of Primary Amino Groups into Other Functional Groups. New Synthetic Methods (41). Angew. Chem. Int. Ed. 1984, 23, 420-429. [CrossRef]

34. Said, S.A.; Fiksdahl, A. Stereoselective Transformation of Amines via Chiral 2,4,6-Triphenylpyridinium Intermediates. Tetrahedron Asymmetry 2001, 12, 1947-1951. [CrossRef]

35. Klauck, F.J.; James, M.J.; Glorius, F. Deaminative Strategy for the Visible-Light-Mediated Generation of Alkyl Radicals. Angezw. Chem. Int. Ed. 2017, 56, 12336-12339. [CrossRef] [PubMed]

36. He, F.-S.; Ye, S.; Wu, J. Recent Advances in Pyridinium Salts as Radical Reservoirs in Organic Synthesis. ACS Catal. 2019, 9 , 8943-8960. [CrossRef]

37. Pang, Y.; Moser, D.; Cornella, J. Pyrylium Salts: Selective Reagents for the Activation of Primary Amino Groups in Organic Synthesis. Synthesis 2020, 52, 489-503.

38. Rössler, S.L.; Jelier, B.J.; Magnier, E.; Dagousset, G.; Carreira, E.M.; Togni, A. Pyridinium Salts as Redox-Active Functional Group Transfer Reagents. Angew. Chem. Int. Ed. 2020, 59, 9264-9280. [CrossRef] [PubMed]

39. Li, Y.-N.; Xiao, F.; Guo, Y.; Zeng, Y.-F. Recent Developments in Deaminative Functionalization of Alkyl Amines. Eur. J. Org. Chem. 2021, 2021, 1215-1228. [CrossRef]

40. Kong, D.; Moon, P.J.; Lundgren, R.J. Radical Coupling from Alkyl Amines. Nat. Catal. 2019, 2, 473-476. [CrossRef]

41. Basch, C.H.; Liao, J.; Xu, J.; Piane, J.J.; Watson, M.P. Harnessing Alkyl Amines as Electrophiles for Nickel-Catalyzed Cross Couplings via C-N Bond Activation. J. Am. Chem. Soc. 2017, 139, 5313-5316. [CrossRef] [PubMed]

42. Hoerner, M.E.; Baker, K.M.; Basch, C.H.; Bampo, E.M.; Watson, M.P. Deaminative Arylation of Amino Acid-Derived Pyridinium Salts. Org. Lett. 2019, 21, 7356-7360. [CrossRef]

43. Liao, J.; Guan, W.; Boscoe, B.P.; Tucker, J.W.; Tomlin, J.W.; Garnsey, M.R.; Watson, M.P. Transforming Benzylic Amines into Diarylmethanes: Cross-Couplings of Benzylic Pyridinium Salts via C-N Bond Activation. Org. Lett. 2018, 20, $3030-3033$. [CrossRef]

44. Baker, K.M.; Baca, D.L.; Plunkett, S.; Daneker, M.E.; Watson, M.P. Engaging Alkenes and Alkynes in Deaminative Alkyl-Alkyl and Alkyl-Vinyl Cross-Couplings of Alkylpyridinium Salts. Org. Lett. 2019, 21, 9738-9741. [CrossRef]

45. Guan, W.; Liao, J.; Watson, M.P. Vinylation of Benzylic Amines via C-N Bond Functionalization of Benzylic Pyridinium Salts. Synthesis 2018, 50, 3231-3237.

46. Plunkett, S.; Basch, C.H.; Santana, S.O.; Watson, M.P. Harnessing Alkylpyridinium Salts as Electrophiles in Deaminative Alkyl-Alkyl Cross-Couplings. J. Am. Chem. Soc. 2019, 141, 2257-2262. [CrossRef]

47. Taylor, B.L.H.; Swift, E.C.; Waetzig, J.D.; Jarvo, E.R. Stereospecific Nickel-Catalyzed Cross-Coupling Reactions of Alkyl Ethers: Enantioselective Synthesis of Diarylethanes. J. Am. Chem. Soc. 2011, 133, 389-391. [CrossRef] [PubMed]

48. Tollefson, E.J.; Dawson, D.D.; Osborne, C.A.; Jarvo, E.R. Stereospecific Cross-Coupling Reactions of Aryl-Substituted Tetrahydrofurans, Tetrahydropyrans, and Lactones. J. Am. Chem. Soc. 2014, 136, 14951-14958. [CrossRef]

49. Marshall, D.R.; Thomas, P.J.; Stirling, C.J.M. Leaving Group Ability in Base-Promoted Alkene-Forming 1,2-Eliminations. J. Chem. Soc. Chem. Commun. 1975, 23, 940-941. [CrossRef]

50. Lucas, E.L.; Hewitt, K.A.; Chen, P.-P.; Castro, A.J.; Hong, X.; Jarvo, E.R. Engaging Sulfonamides: Intramolecular Cross-Electrophile Coupling Reaction of Sulfonamides with Alkyl Chlorides. J. Org. Chem. 2020, 85, 1775-1793. [CrossRef] [PubMed]

51. Steinman, T.J.; Liu, J.; Mengiste, A.; Doyle, A.G. Synthesis of $\beta$-Phenethylmines via Ni/Photoredox Cross-Electrophile Coupling of Aliphatic Aziridines and Aryl Iodides. J. Am. Chem. Soc. 2020, 142, 7598-7605. [CrossRef]

52. Tcyrulnikov, S.; Cai, Q.; Twitty, J.C.; Xu, J.; Atifi, A.; Bercher, O.P.; Yap, G.P.A.; Rosenthal, J.; Watson, M.P.; Kozlowski, M.C. Dissection of Alkylpyridinium Structures to Understand Deamination Reactions. ACS Catal. 2021, 11, 8456-8466. [CrossRef]

53. Xu, J.; Bercher, O.P.; Talley, M.R.; Watson, M.P. Nickel-Catalyzed, Stereospecific C $-\mathrm{C}$ and C $-\mathrm{B}$ Cross-Couplings via C $-\mathrm{N}$ and C-O Bond Activation. ACS Catal. 2021, 11, 1604-1612. [CrossRef]

54. Moragas, T.; Gaydou, M.; Martin, R. Nickel-Catalyzed Carboxylation of Benzylic C-N Bonds with $\mathrm{CO}_{2}$. Angew. Chem. Int. Ed. 2016, 55, 5053-5057. [CrossRef] 
55. Hanessian, S.; Giroux, S.; Mascitti, V. The Iterative Synthesis of Acyclic Deoxypropionate Units and Their Implication in Polyketide-Derived Natural Products. Synthesis 2006, 7, 1057-1076. [CrossRef]

56. Chen, R.; Shen, Y.; Yang, S.; Zhang, Y. Conformational Design Principles in Total Synthesis. Angew. Chem. Int. Ed. 2020, 59, 14198-14210. [CrossRef]

57. Standley, E.A.; Smith, S.J.; Müller, P.; Jamison, T.F. A Broadly Applicable Strategy for Entry into Homogenous Nickel(0) Catalysts from Air-Stable Nickel(II) Complexes. Organometallics 2014, 33, 2012-2018. [CrossRef]

58. Dawson, D.D.; Oswald, V.F.; Borovik, A.S.; Jarvo, E.R. Identification of the Active Catalyst for Nickel-Catalyzed Stereospecific Kumada Coupling Reactions of Ethers. Chem. Eur. J. 2020, 26, 3044-3048. [CrossRef] [PubMed]

59. Tomifuji, R.; Maeda, K.; Takahashi, T.; Kurahashi, T.; Mastubara, S. $\mathrm{FeCl}_{3}$ as an Ion-Pairing Lewis Acid Catalyst. Formation of Highly Lewis Acidic $\mathrm{FeCl}_{2}{ }^{+}$and Thermodynamically Stable $\mathrm{FeCl}_{4}{ }^{-}$To Catalyze the Aza-Diels-Alder Reaction with High Turnover Frequency. Org. Lett. 2018, 20, 7474-7477. [CrossRef]

60. Sabitha, G.; Reddy, N.M.; Prasad, M.N.; Yadav, J.S. Stereoselective Routes for the Total Synthesis of (+)-Crytocarya Diacetate. Helv. Chim. Acta 2009, 92, 967-976. [CrossRef]

61. Tollefson, E.J.; Erickson, L.W.; Jarvo, E.R. Stereospecific Intramolecular Reductive Cross-Electrophile Coupling Reactions for Cyclopropane Synthesis. J. Am. Chem. Soc. 2015, 137, 9760-9763. [CrossRef]

62. Kawana, M. The Reaction of Benzylated Pyrrole and Adenine Ribonucleosides with Grignard Reagents. Chem. Lett. 1981, 10, 1541-1542. [CrossRef]

63. Pöhler, R.; Krahn, J.H.; van den Boom, J.; Dobrynin, G.; Kaschani, F.; Eggenweiler, H.-M.; Zenke, F.T.; Kaiser, M.; Meyer, H. A Non-Competitive Inhibitor of VCP/p97 and VPS4 Reveals Conserved Allosteric Circuits in Type I and II AAA ATPases. Angew. Chem. Int. Ed. 2018, 57, 1576-1580. [CrossRef] [PubMed]

64. Wetterau, J.R.; Gregg, R.E.; Harrity, T.W.; Arbeeny, C.; Cap, M.; Connolly, F.; Chu, C.-H.; George, R.J.; Gordon, D.A.; Jamil, H.; et al. An MTP Inhibitor That Normalizes Atherogenic Lipoprotein Levels in WHHL Rabbits. Science 1998, 282, 751. [CrossRef] [PubMed]

65. Kimura, M.; Masuda, T.; Yamada, K.; Mitani, M.; Kubota, N.; Kawakatsu, N.; Kishii, K.; Inazu, M.; Kiuchi, Y.; Oguchi, K.; et al. Syntheses of Novel Diphenyl Piperazine Derivatives and Their Activities as Inhibitors of Dopamine Uptake in the Central Nervous System. Bioorg. Med. Chem. 2003, 11, 1621-1630. [CrossRef]

66. Dei, S.; Coronnello, M.; Bartolucci, G.; Manetti, D.; Romanelli, M.N.; Udomtanakunchai, C.; Salerno, M.; Teodori, E. Design and synthesis of new potent $\mathrm{N}, \mathrm{N}$-is(arylalkyl)piperazine Derivatives as Multidrug Resistance (MDR) Reversing agents. Eur. J. Med. Chem. 2018, 147, 7-20. [CrossRef] [PubMed]

67. Ameen, D.; Snape, T.J. Chiral 1,1-Diaryl Compounds as Important Pharmacophores. MedChemComm 2013, 4, 893-907. [CrossRef]

68. Yonova, I.M.; Johnson, A.G.; Osborne, C.A.; Moore, C.E.; Morrissette, N.S.; Jarvo, E.R. Stereospecific Nickel-Catalyzed CrossCoupling Reactions of Alkyl Grignard Reagents and Identification of Selective Anti-Breast-Cancer Agents. Angew. Chem. Int. Ed. 2014, 53, 2422-2427. [CrossRef]

69. When (R-BINAP) $\mathrm{NiCl}_{2}$ was employed as the precatalyst, the desired product was not observed and $44 \%$ of starting material was recovered.

70. Chen, P.-P.; Lucas, E.L.; Greene, M.A.; Zhang, S.; Tollefson, E.J.; Erickson, L.W.; Taylor, B.L.; Jarvo, E.R.; Hong, X. A Unified Explanation for Chemoselectivity and Stereospecificity of Ni-Catalyzed Kumada and Cross-Electrophile Coupling Reactions of Benzylic Ethers: A Combined Computational and Experimental Study. J. Am. Chem. Soc. 2019, 141, 5835-5855. [CrossRef]

71. Pangborn, A.B.; Giardello, M.A.; Grubbs, R.H.; Rosen, R.K.; Timmers, F.J. Safe and Convenient Procedure for Solvent Purification. Organometallics 1996, 15, 1518-1520. [CrossRef]

72. Krasoviskiy, A.; Knochel, P. Convenient Titration Method for Organometallic Zinc, Magnesium, and Lanthanide Reagents. Synthesis 2006, 5, 890 .

73. García Ruano, J.L.; Alemán, J.; Belén Cid, M.; Parra, A. A General Method for the Preparation of N-Sulfonyl Aldimines and Ketimines. Org. Lett. 2005, 7, 179-182. [CrossRef]

74. Fu, M.; Chen, L.; Jiang, Y.; Jiang, Z.-X.; Yang, Z. Copper-Catalyzed Intermolecular Chloro- and Bromotrifluoromethylation of Alkenes. Org. Lett. 2016, 18, 348-351. [CrossRef] [PubMed]

75. Syu, S.; Lee, Y.-T.; Jang, Y.-J.; Lin, W. Organocatalytic Tandem Three-Component Reaction of Imine, Alkyl Vinyl Ketone, and Imide via aza-Baylis-Hillman Reaction. J. Org. Chem. 2011, 76, 2888-2891. [CrossRef] [PubMed]

76. Fiorito, D.; Folliet, S.; Liu, Y.; Mazet, C. A General Nickel-Catalyzed Kumada Vinylation for the Preparation of 2-Substituted 1,3-Dienes. ACS Catal. 2018, 8, 1392-1398. [CrossRef]

77. Huang, J.; Zheng, J.; Wu, W.; Li, J.; Ma, J.; Ren, Y.; Jiang, H. Palladium-Catalyzed Intermolecular Oxidative Cyclization of Allyltosylamides with AcOH: Assembly of 3-Pyrrolin-2-ones. J. Org. Chem. 2017, 82, 8191-8198. [CrossRef] [PubMed] 